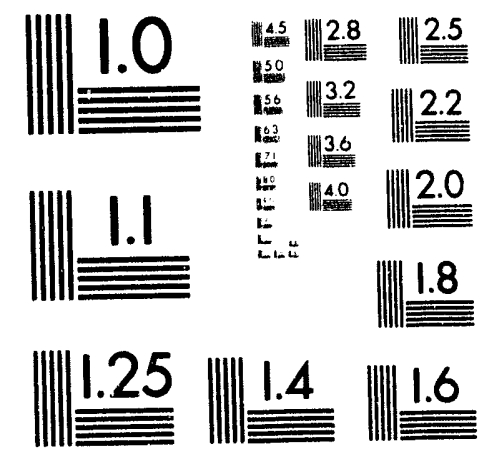



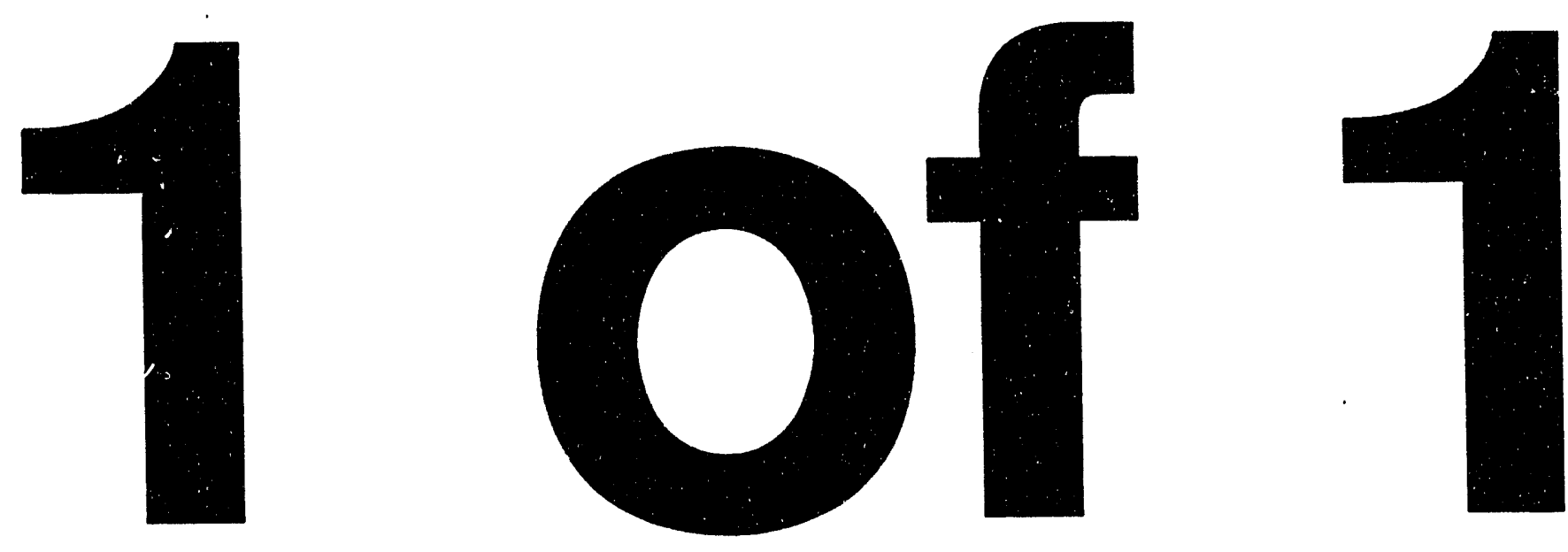


\section{INSTITUTE FOR \\ FUSION STUDIES}

DOE/ET-53088-620

IFSR \#620

Finite Larmor Radius Flute Mode with End Loss

I.A. Kotelnikov

Budker Institute of Nuclear Physics

630090, Novosibirsk, Russia

and

H.L. BERK

Institute for Fusion Studies

The University of Texas at Austin

Austin, Texas 78712

August 1993

\section{THE UNIVERSITY OF TEXAS}

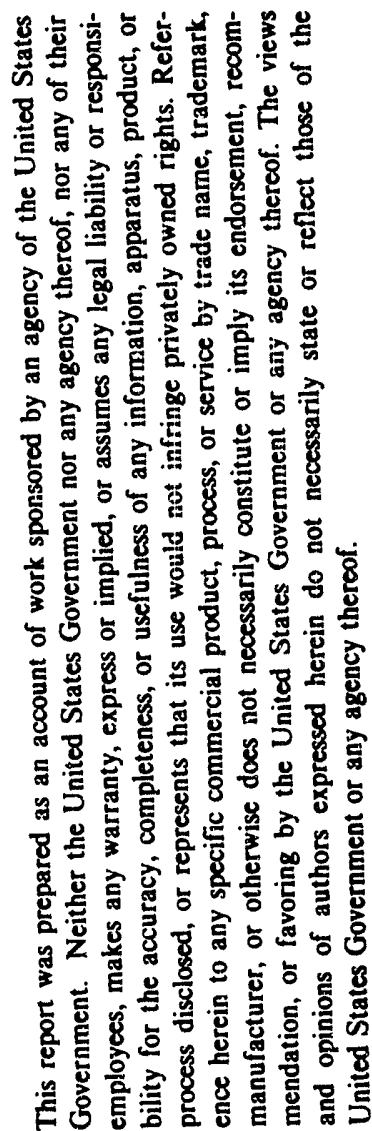




\title{
Finite larmor radius flute mode theory with end loss
}

\author{
I.A. KOTELnikov* and H.L. Berk \\ Institute for Fusion Studies, The University of Texas at Austin \\ Austin, Texas 78712
}

The theory of flute mode stability is developed for a two-energy-component plasma partially terminated by a conducting limiter. The formalism is developed as a preliminary study of the effect of end-loss in open-ended mirror machines where large Larmor radius effects are important.

\section{Introduction}

It is common knowledge that MIID theory predicts that a symmetrically confined mirror configuration is unstable to the flute interchange mode (Rosenbluth and Longmire 1957). The .IHI) stability criterion is altered by finite Larmor radius (FLR) effects (Rosenbluth, Krall, Rostoker 1962) which can stabilize all but the $m=1$ flute mode. The character of the $m=1$ mode that prevents its stabilization is that it is a rigid mode. Under a rigid displacement. the plasma moves as if it is a solid body, so that additional charge separation due to the FLR effect does not appear. An insertion of a conducting limiter does not allow the $m=1$ mode to maintain rigidity because when there is good electrical wall contact the electric potential must drop to zero on the magnetic lines that hit the limiter's surface. Howcver electrical contact of the plasma to the limiter may be too weak to ensure MHD st ability because of low plasma density near the limiter. Attempts to insert the limiter deeper

- Permanent address: Budker Institute of Nuclear Physics, 630090, Novosibirsk, Russia 
into the plasma core has obvious disadvantage as it would degrade plasma conditions. This disadvantage can be by-passed, if a plasma consists of two ion components with significantly different mean energy and energy content. Installing the limiter beyond the region occupied by hot plasma component substantially reduces heat flux to the limiter while relatively cold background plasma can establish electrical contact to the limiter.

In the present paper we develop an electrostatic theory for stability of the very low beta $m=1$ flute mode for a two-component plasma partially terminated by a conducting limiter. In another paper we describe (Berk and Kotelnikov 1993) the relevant results when electromagnetic perturbation are also accounted for at finite beta. Our formalism describes ong going particle flux from the background plasma to the limiter and accounts for FLR effects with the Larmor radius of the hot ions being considered both small and comparable to the plasma radius. Our method of investigation is of interest to such experiments as the Cas-Dynamic Trap (Anikeev et al. 1993) where the end-loss effect, together with finite Larmor radius, is a crucial aspect of the experiment.

The contents of this paper are organized as follows. Section 2 and 3 sketch the Lagrangian thery of .MHD stability appropriate for plasma containing ions with large Larmor radius (i.e. comparable with the plasma radius) (Berk and Wong 1987; Wong, Rosenbluth, Berk 1989). $\operatorname{In} \xi 4$. we give an exact solution to the stability problem of a one-component background plasma terminated by the limiter for arbitrary conditions describing the electrical contact (i.e. arbitrary values for the outgoing particle flux). In the limit of enough large particle los; rate our solution recovers the result of Cohen, Freis, Newcomb (1986) and Liu, Horton, Sedlak (1987) when they consider the boundary condition of zero perturbed potential. Section 5 reformulaies results of $\xi 4$ to the case of the two-component plasma with the Larmor radius of both species being small compared to the plasma radius. In $\S 6$ we present our conclusions. 


\section{Quadratic Variational Form}

For the sake of simplicity we presume that the magnetic field is homogeneous, $\mathbf{B}=B \mathbf{e}_{\mathbf{z}}$. To include the magnetic field curvature, we introduce an artificial radial gravity force. We also allow for the artificial gravity force to have various magnitudes and signs depending on which species it belongs to in order to account for the possibility of different species sampling magnetic field curvature in different ways. Then the equations of the motion take the form

$$
\begin{aligned}
& \ddot{x}=\Omega_{j} \dot{y}-\omega_{j} \Omega_{j} x, \\
& \ddot{y}=-\Omega_{j} \dot{x}-\omega_{j} \Omega_{j} y, \\
& \ddot{z}=0,
\end{aligned}
$$

where $\Omega_{j}=c_{j} B / m_{j} c$ is the cyclotron frequency of a species $j, \omega_{j}=\omega_{\kappa j}+\omega_{E}$,

$$
\omega_{\kappa j}=\overline{\left[\frac{v_{\perp}^{2}}{2}+v_{\|}^{2}\right] \frac{\kappa}{r \Omega_{j}}}
$$

is the drift curvature frequency averaged along a magnetic field line, and

$$
\omega_{E}=\frac{c E}{r H}
$$

is the frequency of the drift caused by the radial equilibrium electric field $E$. In a long-thin (paraxial) system the curvature $\kappa$ is proportional to $r$, thus $\omega_{\kappa j}=$ cunstant. As to the electric drift frequency $\omega_{E}$, its radial behavior depends on many conditions. We consider only the case when $\omega_{E}$ is constant; moreover, we shall entirely neglect the electric drift after we show in $\xi 3.1$ that our derivation reproduces the result of Rosenbluth and Longmire (1957) for a rigidly rotating plasma.

Expressing the position of a particle at a given instant of time $t$ as

$$
\begin{aligned}
& x=r_{G} \cos \theta_{G j}+r_{L} \cos \theta_{L j}, \\
& y=r_{G} \sin \theta_{G j}+r_{L} \sin \theta_{L j},
\end{aligned}
$$


we find from (1) for $r_{G}, r_{L}, v_{\|}$to be constant while the angular and longitudinal coordinates

$$
\begin{gathered}
\theta_{G j}(\tau)=\theta_{G j}+\dot{\theta}_{G j} \tau, \\
\theta_{L j}(\tau)=\theta_{L j}+\dot{\theta}_{L j} \tau, \\
z(\tau)=z+v_{\|} \tau
\end{gathered}
$$

are linear functions of the time $\tau$ reiative to the reference time $t$, with

$$
\begin{aligned}
& \dot{\theta}_{G j}=\frac{1}{2} \Omega_{j}\left[-1+\sqrt{1+4 \omega_{j} / \Omega_{j}}\right] \simeq \omega_{j}-\omega_{j}^{2} / \Omega_{j}, \\
& \dot{\theta}_{L j}=\frac{1}{2} \Omega_{j}\left[-1-\sqrt{1+4 \omega_{j} / \Omega_{j}}\right] \simeq-\Omega_{j}-\omega_{j}
\end{aligned}
$$

being constant. Note that the second term in the right-hand side of (5) can always be neglected for electrons in what follows. This is also true for the ions except for the case when $\omega_{i} \sim \omega$. The latter may occur if there is a sufficiently large radial electric field in the plasma as it is discussed in $\S 3.1$.

The equilibrium distribution is a function of the integrals of motion, i.e. $F_{j}=F_{j}\left(r_{L}, r_{G}, v_{\|}\right)$. In this section, we shall use an alternate set of constants of motion $P_{\theta}=r v_{\theta}+\Omega_{j} r^{2} / 2$, $\mu=v_{\perp}^{2} / 2 B+\omega_{j} \Omega_{j} r^{2} / 2 B$, and $v_{z}$. The two sets are related by the equations

$$
\begin{aligned}
P_{\theta} & =\frac{1}{2} \Omega_{j}\left(r_{G}^{2}-r_{L}^{2}\right) \sqrt{1+\frac{\omega_{j}}{\Omega_{j}}}, \\
\mu & =\frac{\Omega_{j}^{2}}{2 B}\left\{\left[\frac{\omega_{j}}{\Omega_{j}}+\left(\sqrt{\frac{1}{4}+\frac{\omega_{j}}{\Omega_{j}}}+\frac{1}{2}\right)^{2}\right] r_{L}^{2}+\left[\frac{\omega_{j}}{\Omega_{j}}+\left(\sqrt{\frac{1}{4}+\frac{\omega_{j}}{\Omega_{j}}}-\frac{1}{2}\right)^{2}\right] r_{G}^{2}\right\}, \\
v_{z} & =v_{\|} .
\end{aligned}
$$

For any choice of variables, $F_{j}$ is assumed to be normalized so that its first moment gives the unperturbed density of particles of species $j$,

$$
n_{j}(\mathbf{r})=n_{j}(r)=\int d^{3} v F_{j} .
$$


The perturbed distribution function, $f_{j}(\mathbf{r}, \mathbf{v}, t)=f_{j}(r, z, \mathbf{v}) \exp (-i \omega t+i m \theta)$, satisfies the equation

$$
\frac{D f_{j}}{D t}=\frac{e_{j}}{m_{j}} \nabla \phi \cdot \frac{\partial F_{j}}{\partial v}
$$

with $D / D t$ denoting the convective derivative over the unperturbed orbit $(4)$ and $\phi(\mathbf{r}, t)=$ $\widehat{\phi}(r, z) \exp (-i \omega \tau+i m \theta)$ being the perturbed potential. Integrating in time yields

$$
\begin{aligned}
\hat{f}_{j} & =\frac{e_{j}}{m_{j}} \frac{\hat{\phi}}{B} \frac{\partial F_{j}}{\partial \mu}+\frac{e_{j}}{m_{j}} \int_{-\infty}^{0} d \tau \exp [-i \omega \tau+i m(\theta(\tau)-\theta)] \\
& \times\left[i m \frac{\partial F_{j}}{\partial P_{\theta}}+\frac{\partial F_{j}}{\partial v_{z}} \frac{\partial}{\partial z}+\frac{\partial F_{j}}{\partial \mu}\left(i \omega-v_{z} \frac{\partial}{\partial z}\right) \frac{1}{B}\right] \hat{\phi}(r(\tau), z(\tau)),
\end{aligned}
$$

where

$$
\begin{aligned}
r(\tau) & =r(\chi) \equiv \sqrt{r_{G}^{2}+r_{L}^{2}+2 r_{G} r_{L} \cos \chi} \\
\exp (i \theta(\tau)) & =\left[r_{G}+r_{L} \exp (i \chi)\right] \exp \left(i \theta_{G j}(\tau)\right) / r(\tau), \\
\chi & \equiv \theta_{L j}(\tau)-\theta_{G j}(\tau) .
\end{aligned}
$$

We limit our study to the $m=1$ mode and assume that $\partial / \partial z=0$ where the justification is given in Wong, Rosenbluth, Berk (1989). Perfurming the integration in (8), we obtain to lowest order in $\omega / \Omega_{j}$,

$$
\hat{f}_{j}=\frac{\epsilon_{j}}{m_{j}} \frac{\hat{\phi}}{B} \frac{\partial F_{j}}{\partial \mu}-\frac{\epsilon_{j}}{m_{j}}\left\langle\frac{\widehat{\phi}}{r}\left(r_{G}+r_{L} \cos \chi\right)\right\rangle \frac{\exp \left(i \theta_{G j}-i \theta\right)}{\omega-\dot{\theta}_{G j}}\left[\frac{\partial F_{j}}{\partial P_{\theta}}+\frac{\omega}{B} \frac{\partial F_{j}}{\partial \mu}\right],
$$

where the angle brackets $\langle\ldots\rangle$ denote averaging over $\chi$,

$$
\langle\ldots\rangle \equiv \frac{1}{2 \pi} \int_{0}^{2 \pi} d \chi(\ldots)
$$

To avoid possible misunderstanding it is worthwhile to note that the factor $\exp \left(i \theta_{G j}-i \theta\right)$ does not depend on $\theta$ since

$$
\exp \left(i \theta_{G j}-i \theta\right)=\frac{i v_{\tau}-v_{\theta}+r \dot{\theta}_{L j}}{r_{G j}\left(\dot{\theta}_{L j}-\dot{\theta}_{G j}\right)}
$$


The perturbed potential $\phi$ must satisfy the quasi-neutrality equation

$$
\sum_{j} \int d^{3} v e_{j} \widehat{f}_{j} \mathrm{e}^{i \theta}=0
$$

where the index $j$ takes the values $e, i$ for electrons and ions respectively.

Next, we multiply (10) by the adjoint function (Dominguez and Berk 1984) $\phi^{+}=\widehat{\phi}(r) \mathrm{e}^{-i \theta}$ and integrate over all space to produce the quadratic form

$$
L=\sum_{j} \int d^{3} r d^{3} v e_{j} \hat{f}_{j} \hat{\phi}
$$

Transforming the phase space volume integral in (11) to

$$
d^{3} r d^{3} v=\frac{B d \mu d P_{\theta} d v_{z} d \theta d z d \chi}{\dot{\theta}_{L j}-\dot{\theta}_{G j}}
$$

and separating the factors which depend on $x$, we find

$$
\begin{aligned}
L & =\sum_{j} \int 2 \pi B d \mu d P_{\theta} d v_{z} d 0 d z \frac{e_{j}^{2}}{m_{j}\left|\dot{\theta}_{L j}-\dot{\theta}_{G j}\right|} \\
& \times\left[\left\langle\phi^{2}\right\rangle \frac{\partial F_{j}}{B \partial \mu}-\frac{1}{\omega-\dot{\theta}_{G j}}\left\langle\frac{\hat{\phi}}{r}\left(r_{G}+r_{L} \cos \chi\right)\right\rangle^{2}\left(\frac{\partial F_{j}}{\partial P_{\theta}}+\frac{\omega}{B} \frac{\partial F_{j}}{\partial \mu}\right)\right] .
\end{aligned}
$$

Straightforward calculation show's that the variational derivatives $\delta L / \delta \widehat{\phi}$ of (13) is twice lefthand side of (10). Thus, the quadratic form (11) is a self-adjoint quadratic form, i.e. an cxact solution $\hat{\phi}(r)$ of the problem must satisfy to the equations

$$
L=0, \quad \frac{\delta L}{\delta \widehat{\phi}}=0
$$

The alternative quadratic form, $\mathcal{L}$, can be written if we multiply $(10)$ by $\phi^{*}=\widehat{\phi}^{*}(r) \mathrm{e}^{-i \theta}$. This form is not variational, but, when set to zero, it is an identity for the correct eigenfunction and eigenvalue. We note that $\mathcal{L}$ will be useful in stability analysis.

Neglecting any terms which are as small as $\mathcal{O}\left(m_{e} / m_{i}\right)$ we can simplify the electron part $L_{e}$ of $(13)$ to

$$
L_{e}=\int d^{3} r_{G} d^{3} v_{L} \frac{e_{e}^{2}}{m_{e}}\left[-\frac{\omega_{e}}{\omega-\omega_{e}} \frac{\partial F_{e}}{B \partial \mu}-\frac{1}{\omega-\omega_{e}} \frac{\partial F_{e}}{\partial P_{\theta}}\right]\left(\widehat{\phi}\left(r_{G}\right)\right)^{2}
$$


where $d^{3} v_{L} \equiv 2 \pi \Omega_{e}^{2} r_{L} d r_{L} d v_{z}$. Using the equalities

$$
\begin{aligned}
& \frac{\partial F_{e}}{\partial P_{\theta}}=\frac{2}{\Omega_{e}}\left[\frac{\partial F_{e}}{\partial r_{G}^{2}}-\frac{\omega_{e}}{\Omega_{e}} \frac{\partial F_{e}}{\partial r_{L}^{2}}\right], \\
& \frac{\partial F_{e}}{B \partial \mu}=\frac{2}{\Omega_{e}^{2}}\left[\frac{\partial F_{e}}{\partial r_{G}^{2}}+\frac{\partial F_{e}}{\partial r_{L}^{2}}\right],
\end{aligned}
$$

we cast (15) into the form

$$
L_{e}=\int d^{3} r_{G i} d^{3} r_{L} \frac{2 e_{e}^{2}}{m_{e} \Omega_{e}^{2}}\left[-\frac{\Omega_{e}+\omega_{e}}{\omega-\omega_{e}} \frac{\partial F_{e}}{\partial r_{G}^{2}}\right]\left(\hat{\phi}\left(r_{G}\right)\right)^{2} .
$$

With the accuracy $\mathcal{O}\left(m_{e} / m_{i}\right)$ we may neglect $\omega_{e}$ in the numerator of (16). Using $e_{e} n_{e}=$ $-e_{i} n_{i}$ allows to express $L_{e}$ in terms of ion distribution function:

$$
\begin{aligned}
L_{e} & =-\int d^{3} r \frac{2 e_{e}^{2}}{m_{e} \Omega_{e}} \frac{(\widehat{\phi}(r))^{2}}{\omega-\omega_{e}} \frac{\partial}{\partial r^{2}} \int d^{3} v F_{e} \\
& =\int d^{3} r \frac{2 e_{e}^{2}}{m_{e} \Omega_{e}} \frac{(\hat{\phi}(r))^{2}}{\omega-\omega_{e}} \frac{\partial}{\partial r^{2}} \int d^{3} v \frac{e_{i}}{e_{e}} F_{e} \\
& =\int d^{3} r d^{3} v \frac{2 e_{i}^{2}}{m_{i} \Omega_{i}} \frac{(\widehat{\phi}(r))^{2}}{\omega-\omega_{e}} \frac{\partial F_{i}}{\partial r^{2}} .
\end{aligned}
$$

Noting that,

$$
\frac{\partial F_{i}}{\partial r^{2}}=\frac{\Omega_{i}}{2}\left(\frac{r_{G}^{2}+r_{G} r_{L} \cos \chi}{r^{2}}+\frac{\omega_{i}}{\Omega_{i}} \frac{r_{G}^{2}-r_{L}^{2}}{r^{2}}\right) \frac{\partial F_{i}}{\partial P_{\theta}}+\frac{\omega_{i} \Omega_{i}}{2} \frac{\partial F_{i}}{B \partial \mu}
$$

and transforming the space volume integral by means of (12) we find

$$
\begin{aligned}
L= & \int 2 \pi B d \mu d P_{0} d v_{z} d \theta d z \frac{e_{i}^{2}}{m_{i}\left|\dot{\theta}_{L i}-\dot{\theta}_{G i}\right|} \\
& \times\left\{\left[\left(1+\frac{\omega_{i}}{\omega-\omega_{e}}\right)\left\langle\phi^{2}\right\rangle-\frac{\omega}{\omega-\dot{\theta}_{G i}}\left\langle\frac{\hat{\phi}}{r}\left(r_{G}+r_{L} \cos \chi\right)\right\rangle^{2}\right] \frac{\partial F}{B \partial \mu}\right. \\
+ & {\left[\frac{1}{\omega^{\prime}-\omega_{e}}\left\langle\frac{\hat{\phi}^{2}}{r^{2}}\left(r_{C_{i}}^{2}+r_{G} r_{L} \cos \chi\right)\right\rangle-\frac{1}{\omega-\dot{\theta}_{G i}}\left\langle\frac{\hat{\phi}}{r}\left(r_{G}+r_{L} \cos \chi\right)\right\rangle^{2}\right.} \\
& \left.\left.+\frac{\omega_{i} / \Omega_{i}}{\omega-\omega_{e}}\left\langle\frac{\hat{\phi}^{2}}{r^{2}}\left(r_{G}^{2}-r_{L}^{2}\right)\right\rangle\right] \frac{\partial F_{i}}{\partial P_{\theta}}\right\} .
\end{aligned}
$$


We can now substitute $2 \pi B d \mu d P_{\theta} d v_{z} d \theta d z /\left|\dot{\theta}_{L i}-\dot{\theta}_{G i}\right|$ for $d^{3} r_{G} d^{3} v_{L}$. Using the equalities

$$
\begin{aligned}
& \frac{\partial F_{i}}{\partial P_{\theta}}=\frac{2}{\Omega_{i}}\left[\frac{\partial F_{i}}{\partial r_{G}^{2}}-\frac{\omega_{i}}{\Omega_{i}} \frac{\partial F_{i}}{\partial r_{L}^{2}}\right], \\
& \frac{\partial F_{i}}{B \partial \mu}=\frac{2}{\Omega_{i}^{2}}\left[\frac{\partial F_{i}}{\partial r_{G}^{2}}+\frac{\partial F_{i}}{\partial r_{L}^{2}}\right],
\end{aligned}
$$

we find after some amount of algebra that

$$
\begin{aligned}
L= & \frac{2 c_{i}^{2}}{m_{i} \Omega_{i}^{2}} \int_{j} d^{3} r_{G_{i}} d^{3} v_{L}\left\{\left[\left\langle\phi^{2}\right\rangle-\left\langle\frac{\hat{\phi}}{r}\left(r_{G}+r_{L} \cos \chi\right)\right\rangle^{2}\right.\right. \\
& \left.+\frac{\Omega_{i}+2 \omega_{i}}{\omega-\omega_{e}}\left\langle\frac{\hat{\phi}^{2}}{r^{2}}\left(r_{G}^{2}+r_{G} r_{L} \cos \chi\right)\right\rangle-\frac{\Omega_{i}+\omega_{i}}{\omega-\dot{\theta}_{G i}}\left\langle\frac{\hat{\phi}}{r}\left(r_{G}+r_{L} \cos \chi\right)\right\rangle^{2}\right] \frac{\partial F_{i}}{\partial r_{G}^{2}} \\
+ & {\left[\left\langle\phi^{2}\right\rangle-\left\langle\frac{\hat{\phi}}{r}\left(r_{G}+r_{L} \cos \chi\right)\right\rangle^{2}\right.} \\
& \left.\left.+\frac{\omega_{i}}{\omega-\omega_{e}}\left(\left\langle\hat{\phi}^{2}\right\rangle-\left\langle\frac{\hat{\phi}^{2}}{r^{2}}\left(r_{G}^{2}+r_{G} r_{L} \cos \chi\right)\right\rangle\right)\right] \frac{\partial F_{i}}{\partial r_{L}^{2}}\right\}
\end{aligned}
$$

where in $\hat{\phi}=\widehat{\phi}(r)$ we use $r=\left(r_{G}^{2}+r_{L}^{2}+2 r_{L} r_{G} \cos \chi\right)^{1 / 2}$. This quadratic form accounts for the axis encircling ions, $r_{G}<r_{L}$, as we have not treated these particles any differently from ions where $r_{G}>r_{L}$. The difference $\dot{\theta}_{G i}$ from $\omega_{G i}$, as it was pointed out when we discussed the (5), will be taken into account only in the case of a rotating plasma which is only considered in the next section.

\section{Reduction of Quadratic Form}

When the Larmor radius is small the quadratic form (19) can be substantially simplified by means of expanding the quantities in the angular brackets in a Taylor series near the point $r(\chi)=r_{G}$ provided that $r_{L} \ll r_{G}$. For example,

$$
\left\langle\widehat{\phi}^{2}\right\rangle \simeq \widehat{\phi}^{2}+\langle\delta r\rangle\left(\hat{\phi}^{2}\right)^{\prime}+\frac{1}{2} \delta r^{2}\left(\hat{\phi}^{2}\right)^{\prime \prime}
$$


where $\delta r=r(\chi)-r_{G}, \hat{\phi}$ in the right-hand-side is to be taken at $r_{G}$, and the prime denotes differentiation over $r_{G}$. It is readily to show that $\langle\delta r\rangle=r_{L}^{2} / 4 r_{G},\langle\delta r\rangle^{2}=r_{L}^{2} / 2$, so that

$$
\left\langle\widehat{\phi}^{2}\right\rangle=\widehat{\phi}^{2}+A+\frac{r_{L}^{2}}{2}\left[\hat{\phi}^{\prime 2}+\frac{\widehat{\phi}^{2}}{r_{G}}\right]
$$

where

$$
A=\frac{r_{L}^{2}}{2}\left[3 \hat{\phi}\left(\frac{\hat{\phi}}{r_{G}}\right)^{\prime}+r_{G} \hat{\phi}\left(\frac{\hat{\phi}}{r_{G}}\right)^{\prime \prime}\right] .
$$

Repeating the calculation for other brackets gives

$$
\begin{aligned}
\left\langle\frac{\hat{\phi}}{r}\left(r_{G}+r_{L} \cos \chi\right)\right\rangle^{2} & =\widehat{\phi}^{2}+A \\
\left\langle\frac{\hat{\phi}^{2}}{r^{2}}\left(r_{G}^{2}+r_{G} r_{L} \cos \chi\right)\right\rangle & =\hat{\phi}^{2}+A+\frac{r_{L}^{2}}{2}\left(r_{G}\left(\frac{\hat{\dot{\varphi}}}{r_{G}}\right)^{\prime}\right)^{2} .
\end{aligned}
$$

\subsection{Rotating plasma}

In order to compare our results with that of Rosenbluth, Krall, and Rostoker (1962) we first consider a specific case of a plasma rotating with a frequency $\omega_{E}$ as a rigid body due to, say, an equilibrium electric field, $\omega_{E}=c E / r H=$ const If the electron and ion temperatures are essentially of the same order of magnitude, $T_{e} \sim T_{i}$, then the electric drift frequency can be estimated as $\omega_{E} \sim\left(\rho_{i} / r_{0}\right)^{2} \Omega_{i}$ where $r_{0}$ denotes the plasma radius, and $\rho_{i}$ denotes mean ion Larmor radius. We shall show (see also (Rosenbluth and Longmire 1957)), that FLR effects play a significant role provided that $\kappa r_{0} \sim \rho_{i}^{2} / r_{0}^{2}$. For these conditions we have $\omega_{\kappa e} \sim \omega_{\kappa i} \sim\left(\rho_{i} / r_{0}\right)^{4} \Omega_{i}$ and, hence, the curvature drift is negligible in comparison with the electric drift, $\omega_{\kappa e} \sim \omega_{\kappa i} \ll \omega_{E}$. Therefore we can set $\omega_{\kappa e}=\omega_{\kappa i}=0$ and

$$
\dot{\theta}_{G e}=\omega_{E}, \quad \dot{\theta}_{G i}=\omega_{E}-\omega_{E}^{2} / \Omega_{i}
$$

Using the ordering scheme $\omega \sim \omega_{E} \sim\left(r_{L} / r_{0}\right)^{2} \Omega_{i}$ reduces (19) to

$$
L_{\mathrm{r}, \mathrm{t}}=\frac{2 e_{i}^{2}}{m_{i} \Omega_{i}^{2}} \int d^{3} r_{C_{i}} d^{3} v_{L}\left\{\frac{r_{L}^{2}}{2}\left[\frac{\omega}{\omega-\omega_{E}}\left(\left(\hat{\phi}^{\prime}\right)^{2}+\left(\frac{\hat{\phi}}{r_{G}}\right)^{2}\right)-\frac{\omega_{E}}{\omega-\omega_{E}}\left(r_{G}\left(\frac{\hat{\phi}}{r_{G}}\right)^{\prime}\right)^{2}\right] \frac{\partial F_{i}}{\partial r_{L}^{2}}\right.
$$




$$
\left.+\left[\frac{\omega \omega_{E}}{\left(\omega-\omega_{E}\right)^{2}} \widehat{\phi}^{2} \quad+\frac{r_{L}^{2}}{2} \frac{\Omega_{i}}{\omega-\omega_{E}}\left(r_{G}\left(\frac{\hat{\phi}}{r_{G}}\right)^{\prime}\right)^{2}\right] \frac{\partial F_{i}}{\partial r_{G}^{2}}\right\} .
$$

For Maxwellian distribution of the ions with homogeneous temperature, $\partial T_{i} / \partial r=0, L_{\text {rot }}$ reduces to

$$
\begin{aligned}
L_{\mathrm{rot}} & =\frac{e_{i}^{2}}{m_{i} \Omega_{i}^{2}} \int d^{3} r\left\{-\left[\frac{\omega}{\omega-\omega_{E}}\left(\left(\hat{\phi}^{\prime}\right)^{2}+\left(\frac{\widehat{\phi}}{r}\right)^{2}\right)-\frac{\omega_{E}}{\omega-\omega_{E}}\left(r\left(\frac{\hat{\phi}}{r}\right)^{\prime}\right)^{2}\right] n_{i}\right. \\
& \left.+\left[\frac{\omega \omega_{E}}{\left(\omega-\omega_{E}\right)^{2}} \hat{\phi}^{2}+\frac{\rho_{i}^{2}}{2} \frac{\Omega_{i}}{\omega-\omega_{E}}\left(\left(\frac{\widehat{\phi}}{r}\right)^{\prime}\right)^{2}\right] \frac{\partial n_{i}}{r \partial r}\right\}
\end{aligned}
$$

where $\rho_{i}$ is the average Larmor radius of the background ions, and $r$ is used to substitute $r_{G}$ because after the expansion being carried out any difference between $r_{G}$ and $r$ are negligible. The terms in the first square brackets in (20) may be thought to represent the inertia of the plasma, while the two terms in the second square brackets are responsible for the rotational drive of the instability and FLR effects respectively. Varying the quadratic form for the Ciaussian density profile $\dot{n}_{2}(r)=n_{0} \exp \left(-r^{2} / r_{0}^{2}\right)$ with $r_{0}$ being the plasma radius, yields the equation

$$
\begin{gathered}
\widehat{\phi}^{\prime \prime}+\frac{1}{r} \widehat{\phi}^{\prime}-\frac{1}{r^{2}} \widehat{\phi}-\frac{2 r}{r_{i}^{2}} \widehat{\phi}^{\prime}+\frac{2 \lambda}{r_{0}^{2}} \widehat{\phi}=0, \\
\lambda=\left[\frac{\left(\rho_{i} / r_{0}\right)^{2} \Omega_{i}-2 \omega_{E}}{\omega-\omega_{E}}-\frac{\omega_{E}^{2}}{\left(\omega-\omega_{E}\right)^{2}}\right] /\left[1+\frac{\left(\rho_{i} / r_{0}\right)^{2} \Omega_{i}}{\omega-\omega_{E}}\right]
\end{gathered}
$$

which coincides with (3.19) from the paper of Rosenbluth, Krall, and Rostoker (1962).

\subsection{Bacíground plasma}

In the rest of the paper, we neglect the radial electric field (e.g. this is natural if $T_{e} \ll T_{i}$ ), hence $\omega_{E}:=0$, and

$$
\dot{\theta}_{G e}=\omega_{\kappa e}, \quad \dot{\theta}_{G i}=\omega_{\kappa i} .
$$

Assuming the FLR effects to be strong enough, i.e.

$$
\omega_{r i} \sim \omega_{r e} \sim\left(\rho_{i}^{2} / r_{0}^{2}\right)^{2} \Omega_{i} .
$$


we find that the frequency of the flute mode $\omega \sim \sqrt{\omega_{\kappa i, e} \Omega_{i}}$ is of order $\left(\rho_{i}^{2} / r_{0}^{2}\right) \Omega_{i}$. With this ordering, we obtain

$$
L_{c}=\frac{e_{i}^{2}}{m_{i} \Omega_{i}^{2}} \int d^{3} r\left\{-\left[\hat{\phi}^{\prime 2}+\left(\frac{\hat{\phi}}{r}\right)^{2}\right] n_{i}-\left[\frac{\left(\omega_{\kappa i}-\omega_{\kappa e}\right) \Omega_{i}}{\omega^{2}} \hat{\phi}^{2}-\frac{\rho_{i}^{2}}{2} \frac{\Omega_{i}}{\omega}\left(r\left(\frac{\hat{\phi}}{r}\right)^{\prime}\right)^{2}\right] \frac{\partial n_{i}}{r \partial r}\right\} .
$$

The first square bracket in (22) represents the inertia of the plasma. The first term in the second square bracket is the curvature drive, and the last term describes FLR effects. Varying $L_{c}$ over $\hat{\phi}$ reproduces the same equation (21) with the redefined coefficient

$$
\lambda=1-\frac{1-\left(\omega_{\kappa i}-\omega_{\kappa e}\right) \Omega_{i} / \omega^{2}}{1+\left(\rho_{i} / r_{0}\right)^{2} \Omega_{i} / \omega}
$$

which coincides with (3.27) from Rosenbluth and Longmire (1957).

\subsection{Hot-ion-plasma}

Now we take the plasma to contain a hot ion component which we label with the subscript $h$ to distinguish it from the relatively cold background ions for which we keep the label $i$. Because of high energy drift frequency of hot ions is much greater than those of electrons and background ions, $\omega_{\kappa h} \gg \omega_{\kappa i} \sim \omega_{\kappa e}$. For now we take the average Larmor radius $\rho_{h}$ of the hot ions to be comparable with the plasma radius $r_{0}$ and thus keep the angle brackets in (19) unexpanded. Nevertheless the quadratic form can still be simplified after specifying the ratio of the hot density $n_{h}$ to the background one $n_{i}$. In a pure hot-ions-plasma, $n_{i} \ll n_{h}$, the flute mode has a frequency $\omega$ close to $\sqrt{\omega_{\kappa h} \Omega_{h}}$ so that (11) takes the form

$$
\begin{aligned}
L= & \frac{2 c_{h}^{2}}{m_{h} \Omega_{h}^{2}} \int d^{3} r_{G} d^{3} v_{L}\left\{\left[\left\langle\phi^{2}\right\rangle-\left\langle\frac{\hat{\phi}}{r}\left(r_{G}+r_{L} \cos \chi\right)\right\rangle^{2}\right] \frac{\partial F_{h}}{\partial r_{L}^{2}}\right. \\
+ & {\left[\left\langle\phi^{2}\right\rangle-\left\langle\frac{\hat{\phi}}{r}\left(r_{G}+r_{L} \cos \chi\right)\right\rangle^{2}-\frac{\omega_{x h} \Omega_{h}}{\omega^{2}}\left\langle\frac{\hat{\phi}}{r}\left(r_{G}+r_{L} \cos \chi\right)\right\rangle^{2}\right.} \\
& \left.\left.+\frac{\Omega_{h}}{\omega}\left(\left\langle\frac{\hat{\phi}^{2}}{r^{2}}\left(r_{G}^{2}+r_{G} r_{L} \cos \chi\right)\right\rangle-\left\langle\frac{\hat{\phi}}{r}\left(r_{G}+r_{L} \cos \chi\right)\right\rangle^{2}\right)\right] \frac{\partial F_{h}}{\partial r_{G}^{2}}\right\} .
\end{aligned}
$$


Here the terms which do not depend on the frequency can be interpreted as an inertia of the plasma while the term with the factor $\omega_{\kappa h} \Omega_{h} / \omega^{2}$ describes the curvature drive for the flute instability. The last two terms, proportional to $\Omega_{h} / \omega$, are responsible for FLR stabilization. It is readily shown that they cancel each other if the mode is rigid, i.e. $\hat{\phi}(r)=\left(r / r_{0}\right) \phi_{0}$. Typically such an eigenfunction structure is accurate, when $r_{0}^{2} \omega_{\kappa h} / \omega \rho_{h}^{2}>1$, as the FLR terms are typically much greater than the other terms except when the eigenfunctions are nearly linear in $r$. Therefore the flute mode must be almost rigid. For the exact rigid mode (23) becomes

$$
L=\frac{2 e_{h}^{2}}{m_{h} \Omega_{h}^{2}} \frac{\phi_{0}^{2}}{r_{0}^{2}}\left(\frac{\omega_{\kappa h} \Omega_{h}}{\omega^{2}}-1\right) N_{h}
$$

where $V_{h}=\int d^{3} r_{G} d^{3} v_{L} V_{h}$ is the total number of hot ions, and the identity

$$
\int d^{3} r_{G} \partial F_{h} / \partial r_{G}^{2}=\left.\int d z F_{h}\right|_{r_{G}=-0} ^{r_{G}=\infty}=0
$$

has been used. Equating (24) to 0 yields $\omega=\sqrt{\omega_{\kappa h} \Omega_{h}}$.

\subsection{Two-component plasma}

Let us now consider a two-component plasma produced by injecting high-energy neutral atoms into a relatively cold dense plasma core. In such a case, the hot ions comprise a small fraction of the plasma density, $n_{h} \ll n_{i} \simeq n_{e}$, but may overwhelm the pressure of the background plasma. More precisely we assume that $n_{h} / n_{i} \sim \omega_{\kappa h} / \Omega_{h} \gg\left(\rho_{i} / \rho_{h}\right)^{2}$. Then the frequency $\omega$ of the mode under investigation turns out to be of order $\omega_{\kappa h}$. Based on these assumptions we keep the contribution of the background plasma $L_{c}$ to the quadratic form $L$ in the form (22) but neglect the inertia of the hot ions so that

$$
\begin{aligned}
L_{\mathrm{h}}= & \frac{2 e_{h}^{2}}{m_{h} \Omega_{h}^{2}} \int d^{3} r_{G} d^{3} v_{L} \times \\
& {\left[\frac{\Omega_{h}}{\omega}\left\langle\frac{\hat{\phi}^{2}}{r^{2}}\left(r_{G}^{2}+r_{G} r_{L} \cos \chi\right)\right\rangle-\frac{\Omega_{h}}{\omega-\omega_{\kappa h}}\left\langle\frac{\hat{\phi}}{r}\left(r_{G}+r_{L} \cos \chi\right)\right\rangle^{2}\right] \frac{\partial F_{h}}{\partial r_{G}^{2}} . }
\end{aligned}
$$




\subsection{Outgoing particle flow}

The conducting limiter affects the plasma stability by means of the outgoing current it collects from the background plasma while the ions of hot species are presumed to be isolated from the limiter by the magnetic mirror. To describe the conditions near the limiter surface, we assume the background plasma to be Maxwellian and no net current is drawn by the limiter in the equilibrium because a Debye sheath that maintains overall quasineutrality, is formed close to the surface. In other words, the drop of potential $\Phi$ through the sheath makes an electron longitudinal (parallel to $\mathbf{B}$ ) current

$$
j_{\| e}=\frac{e_{e} n}{\sqrt{\pi} v_{e}} \int_{\sqrt{-2 e_{e} \Phi / m_{e}}}^{\infty} d v_{\|} v_{\|} \exp \left(-\frac{v_{\|}^{2}}{v_{e}^{2}}\right)=\frac{e_{e} n v_{e}}{2 \sqrt{\pi}} \exp \left(\frac{e_{e} \Phi}{T_{e}}\right)
$$

that just balances the ion current

$$
j_{\| i}=\epsilon \frac{e_{i} n}{\sqrt{\pi} v_{i}} \int_{0}^{\infty} d v_{\|} v_{\|} \exp \left(-\frac{v_{\|}^{2}}{v_{i}^{2}}\right)=\epsilon \frac{e_{i} n v_{i}}{2 \sqrt{\pi}}
$$

at the limiter's surface, and in equilibrium $j_{\| e}+j_{\|_{i}}=0$ at $\Phi=\Phi_{0}$. Here $v_{i, e}=\sqrt{2 T_{i, e} / m_{i, e}}$ is the thermal velocity, $n$ and $\Phi$ are the plasma density and potential outside the sheath (potential of the limiter is chosen to be zero), and $\epsilon \leq 1$ is a phenomenological parameter to describe the effect of a large end mirror and it stands for the fraction of ions absorbed by the limiter from total influx (e.g. see Berk, Ryutov, Tsidulko (1991)). The rest of the ion flux is assumed to be reflected. In what follows we assume that the reflection coefficient is a function of radius, $\epsilon=\epsilon(r)$, which nearly vanishes if $r$ is smaller than the inner radius $r_{*}$ of the limiter. The perturbed current is easy to find from (26) and (27).

$$
\delta j_{\| e}=-j_{\|_{i}} e_{e} \phi / T_{e}-\delta n_{i} j_{\|_{i}} / n_{i} ; \quad \delta j_{\|_{i}}=\delta n_{i} j_{\|_{i}} / n_{i}
$$

We now incorporate the loss term into the quadratic form in a manner that retains the variational structure. The change, $\phi$, in the plasma potential $\Phi=\Phi_{0}+\phi$ causes a change 
in the outgoing current $\delta \mathbf{j}=\left(\delta j_{\| \mathbf{i}}+\delta j_{\| e}\right) \mathbf{b}$, which in turn causes an additional change in the charge density

$$
e_{j} \delta n_{j}=-\frac{i}{\omega} \operatorname{div} \delta j_{\| \mathbf{i}} \mathbf{b}, \quad j=e, i .
$$

Here $\mathbf{b}=\mathbf{B} / B$. We assume that the response of the original terms, which is due only to the perturbed perpendicular currents is unaltered by particle loss. The approximation is accurate if $\omega T \gg 1$, where $T$ is the lifetime of particles on the lossy field lines. The additional perturbed density due to end loss causes an additional quadratic term,

$$
L_{1}=\sum_{j} \int d^{3} r e_{j} \delta n_{j} \phi^{+}
$$

which needs to be added to the quadratic form $L$. This new term can be transformed to the integral over the surface of the limiter as $\phi^{+}$is independent of the axial coordinate,

$$
L_{1}=-\frac{i}{\omega} \sum_{j} \oint d \mathbf{S} \cdot \mathbf{b} \delta j_{\| j} \phi^{+}
$$

with the potential $\phi$ being taken on the plasma side of the Debye's sheath. Assuming the limiter's surface to be perpendicular to the direction of the magnetic field we obtain

$$
L_{1}=-i \frac{\Omega_{i}}{\omega} \frac{e_{i}^{2}}{m_{i} \Omega_{i}^{2}} \int_{0}^{\infty} d r 2 \pi r \frac{k \epsilon(r)}{\sqrt{\pi} \rho_{i}}\left|\frac{e_{e}}{e_{i}}\right| \frac{T_{i}}{T_{e}} n_{i}(r) \hat{\phi}^{2}
$$

where $k=1,2 \ldots$ is the number of absorbing surfaces of the limiter(s). The external radius of the radial liner surrounding the plasma column is assumed to be sufficiently large in comparison with the plasma radius $r_{0}$ so that none of the particles terminate on it. In the next sections, we shall omit the factor $k\left|e_{e} / e_{i}\right|\left(T_{i} / T_{e}\right)$ assuming it to be included into the redefined reflection coefficient $\epsilon$.

The sum of the combined terms, $L+L_{1}$, has a variational structure. The variation with respect to $\phi$ gives the equation

$$
\frac{\partial L}{\partial \phi}+\frac{\partial L_{1}}{\partial \phi}=0
$$

where $\partial L / \partial \phi$ is the perturbed density without particle loss, and $\partial L_{1} / \partial \phi$ is identical to the particle leakage term described in other theories (e.g. (Berk, Ryutov, Tsidulko 1991)). 


\section{The Effect of Limiter on Flute Stability}

To give an idea how a conducting limiter affects plasma stability, we, first, consider a background plasma without hot ions. With high degree of reliability, its density can be assumed to have the diffuse (Gaussian) profile, $n(r)=n_{0} \exp \left(-r^{2} / r_{0}^{2}\right)$, except for the shadow region $r>r_{*}$ terminated by the limiter's surface; beyond the limiter's edge the plasma density drops rapidly almost to zero in a short distance $\Delta r$ (see figure 1).

It is obvious that perfect electrical contact between the plasma and the limiter prevents any finite perturbation of the electric potential $\phi$ in the shadow of the limiter (i.e. at the magnetic field lines which match the limiter's surface). The solution of the appropriate boundary-value problem comprising (21) with zero boundary condition, $\hat{\phi}=0$ at $r=r_{*}$, has been considered by others (Cohen, Freis, Newcomb 1986; Liu, Horton, Sedlak 1y87; Anikeev, Bagryansky, Ivanov, Kuzmin, Salikova 1992) and it causes stabilization by FLR effects if $r_{*}$ is sufficiently small. In the opposite case of electrically isolated limiter, the perturbation for the $m=1$ becomes rigid. Our goal here is to track the transformation of the rigid flute mode into the "stabilized" one.

Appropriate to the problem under discussion is the quadratic form for only the background plasma, which now takes the form $L_{\mathrm{cl}} \equiv L_{\mathrm{c}}+L_{1}$. We rewrite it with the dimensionless magnetic flux $\psi$ defined as $\psi=r^{2} / r_{0}^{2}$ and with the new function $g(\psi)$ as $g(\psi)=\widehat{\phi} /\left(\phi_{0} \sqrt{\psi}\right)$. Then $L_{\mathrm{cl}}$ has the form,

$$
\begin{aligned}
L_{\mathrm{cl}}= & \frac{\pi e_{i}^{2} \phi_{0}^{2} l}{m_{i} \Omega_{i}^{2}} \int_{0}^{\infty} d \psi \times \\
& \left\{-\left[g^{2}+\left(g+2 \psi g^{\prime}\right)^{2}\right] n_{i}+4 \psi^{2} g^{\prime 2} n_{i}^{\prime} \frac{\omega_{*_{i}}}{\omega}+2 \psi g^{2} n_{i}^{\prime} \frac{\Gamma^{2}}{\omega^{2}}-2 i \psi g^{2} n_{i} \frac{\nu}{\omega}\right\},
\end{aligned}
$$

where $g, n_{i}$, and $\epsilon$ are functions of $\psi, \Gamma^{2} \equiv-\left(\omega_{\kappa i}-\omega_{\kappa e}\right) \Omega_{i}$ is the square of the MHD growth rate, $\omega \boldsymbol{*}_{i} \equiv\left(\rho_{i} / r_{0}\right)^{2} \Omega_{i}$ is the diamagnetic frequency, $\nu=\nu(\psi) \equiv(\epsilon(\psi) / 2 \sqrt{\pi})\left(r_{0}^{2} / l \rho_{i}\right) \Omega_{i}$ is the effective leakage frequency, $l$ denotes the length of the plasma column, and the prime stands 
for the derivative with respect to $\psi$. The proper variational equation is

$$
\left[\psi^{2}\left(n_{i}-\frac{\omega_{*_{i}}}{\omega} n_{i}^{\prime}\right) g^{\prime}\right]^{\prime}+\frac{1}{2} \psi\left[\left(1+\frac{\Gamma^{2}}{\omega^{2}}\right) n_{i}^{\prime}-i \frac{\nu}{\omega} n_{i}\right] g=0 \text {. }
$$

Due to leakage of the background plasma onto the limiter surfaces, the density drop in the scrape-off layer varies sharply, which makes the density gradient inside the scrape-off layer much larger than in the region not shadowed by the limiter, i.e. where $\psi<\psi_{*}=\left(r_{*} / r_{0}\right)^{2}$. Therefore $n_{i}^{\prime}(\psi)$ is not continuous at $\psi=\psi_{*}$.

The exact position of the plasma-vacuum boundary $\psi=\psi_{*}+\Delta \psi$ is not essential for what follows. For the sake of clarity, we can place it at such a flux where $n_{i}=0$, while $n_{i}^{\prime} \neq 0$ at the plasma side of the boundary though $n_{i}^{\prime}=0$ at the vacuum side.

The boundary conditions are readily derived from (30). Integrating it over a thin layer surrounding a boundary yields that

$$
\left.\left(n_{i}-\frac{\omega_{*_{i}}}{\omega} n_{i}^{\prime}\right) g^{\prime}\right|_{\psi_{*}^{-}} ^{\psi_{*}^{+}}=0 ;\left.\quad g\right|_{\psi_{*}^{-}} ^{\psi_{*}^{+}}=0 .
$$

Assuming the FLR effects to be essential so that $\omega \sim \omega_{*_{i}}$ (see $\S 3.3$ ), we find from (31) that $g^{\prime} \Delta \psi \ll 1$ inside the scrape-off layer since this conditions holds on both sides of the layer. As we shall see, this condition breaks only if the flute mode is very strongly stabilized; an exact critcrion will be given later. Hence, to track the above mentioned transformation between rigid and "stabilized" modes, $g(\psi)$ may be taken constant across the density drop. Integrating $(30)$ from $\psi_{*}^{-}$to $\psi_{*}+\Delta \psi^{+}$with constant $g(\psi)=g\left(\psi_{*}\right)$ yields the integral boundary condition

$$
\psi_{*} \frac{g^{\prime}}{g}=-\frac{1+\Gamma^{2} / \omega^{2}+i \nu \Delta \psi / \omega}{2\left[1-\left(\omega_{*_{i}} / \omega\right)\left(n_{i}^{\prime} / n_{i}\right)\right]}
$$

where $g^{\prime} / g$ and $n_{i}^{\prime} / n_{i}$ is associated with the inner plasma side of the boundary $\psi=\psi_{*}$, while the leakage frequency $\nu$ belongs to the steep gradient region (where $\nu$ is constant), and

$$
\Delta \psi=\int_{\psi_{*}}^{\infty} d \psi \frac{n_{i}(\psi)}{n_{i}\left(\psi_{*}\right)}=\int_{r_{*}}^{\infty} \frac{d r 2 r}{r_{0}^{2}} \frac{n_{i}(r)}{n_{i}\left(r_{*}\right)} .
$$


is the effective width of the gradient region.

In the region $\psi<\psi *$ where $n_{i}(\psi)=n_{\mathfrak{i} 0} \exp (-\psi)$ and $\nu(\psi)=0$. The differential equation in this region is satisfied by the confluent hypergeometric (Kummer) function:

$$
g(\psi)={ }_{1} F_{1}(a, 2 ; \psi)
$$

where

$$
a(\omega) \equiv \frac{1}{2} \frac{\left(1+\Gamma^{2} / \omega^{2}\right)}{1+\omega *_{i} / \omega} .
$$

When substituted into the left-hand side of (32), it produces the dispersion relation

$$
\psi_{*} \frac{{ }_{1} F_{1}^{\prime}\left(a(\omega), 2 ; \psi_{*}\right)}{{ }_{1} F_{1}\left(a(\omega), 2 ; \psi_{*}\right)}=-a(\omega)-i q(\omega)
$$

where

$$
q(\omega) \equiv \frac{1}{2} \frac{\nu \Delta \psi / \omega}{1+\omega_{*_{i}} / \omega},
$$

and prime denotes derivative over $\psi_{*}$. Using asymptotics of the Kummer's function for $a \rightarrow 0$ and $\psi \gg 1$

$$
{ }_{1} F_{1}(a, 2 ; \psi) \simeq 1+\frac{a}{\psi^{2}} \exp (\psi),
$$

we assume $a \ll 1$ and we can approximate the dispersion relation as

$$
a(\omega) \equiv-\frac{i q(\omega) / \psi_{*}}{1+i q(\omega) / \psi_{*}} a_{*}
$$

where

$$
a_{*} \equiv \psi_{*}^{2} \exp \left(-\psi_{*}\right)=\left(r_{*} / r_{0}\right)^{4} \exp \left(-r_{*}^{2} / r_{0}^{2}\right)
$$

Note that $a(\omega)$ is exponentially small and thus even for a mild ratio $r_{*} / r_{0} \simeq 2$ our asymptotic approximation is accurate. Another assumption, which is the near constancy of $g(\psi)$ inside the scrape-off layer, uses the condition

$$
q / \psi_{*} \sim \nu_{*} /\left(\omega+\omega_{*_{i}}\right) \ll 1 / \Delta \psi^{2} .
$$


where we have introduced the notation

$$
\nu_{*}=\nu \Delta \psi / 2 \psi_{*} \sim(\epsilon / 4 \sqrt{\pi})\left(r_{0}^{4} / l \rho_{i}^{3}\right)\left(\Delta \psi / \psi_{*}\right) \omega_{*_{i}}
$$

useful for what follows. If (40) is not the case, the integral boundary condition (32) can not be used. Instead, the function $g(\psi)$ inside the scrape-off layer is to be found from (30) and then matched to (34) with the primary boundary conditions (31). For the extreme case

$$
\nu_{*} /\left(\omega+\omega_{*_{i}}\right) \gg 1 / \Delta \psi^{2}
$$

(30) becomes

$$
g^{\prime \prime}-2 i \lambda^{2} g=0
$$

with

$$
\lambda^{2}(\psi)=-\frac{\nu}{4 \omega_{*_{i}}} \frac{n_{i}(\psi)}{\psi n_{i}^{\prime}(\psi)} .
$$

Since the inequality ( 11$)$ means that $\lambda^{2} / \lambda^{\prime} \gg 1,(42)$ can be solved by using the quasiclassical approach. Keeping only the damping exponent gives

$$
g(\psi)=g\left(\psi_{*}\right) \exp \left[-(1+i) \int_{\psi_{*}}^{\psi} d \psi \lambda(\psi)\right]
$$

The solution with the growing exponent is discarded because $\int_{\psi_{*}}^{\psi_{*}+\Delta \psi} d \psi \lambda(\psi) \gg 1$. Matching (43) with (34) by means of (31) yields

$$
\dot{\psi}_{*} \frac{{ }_{1} F_{1}{ }^{\prime}\left(a(\omega), 2 ; \psi_{*}\right)}{{ }_{1} F_{1}} \frac{\left(1+i, ? ; \psi_{*}\right)}{(a) \omega_{*} / \omega} \sqrt{\frac{\nu}{2\left(1+\omega_{*_{i}} / \omega\right)} \frac{\psi_{*}}{\Delta \psi_{* i}}}
$$

where $\Delta \psi_{*}^{\prime}$ stands for $-n_{i}\left(\psi_{*}\right) / n_{i}^{\prime}\left(\psi_{*}+0\right)$. This relation can also be cast into an asymptotic form as was done in obtaining (39). However we avoid further details at this point because the transition between the rigid and stabilized flute modes occurs at $q / \psi_{*} \sim 1$ when the inequality (40) holds.

The scrape-off layer has a negligible effect if $q / \psi_{*} \ll 1$ (i.e. $\nu_{*} /\left(\omega_{*_{i}}+\omega\right) \ll 1$ ), as then the dispersion relation (39) becomes

$$
a(\omega)=0
$$


which gives the rigid mode $\left(g^{\prime}=0\right)$ dispersion relation,

$$
\omega_{1,2}= \pm i \Gamma
$$

It is unstable if $\Gamma^{2}>0$.

In the opposite case, $q / \psi_{*} \gg 1$ (i.e. $\nu_{*} /\left(\omega_{*_{i}}+\omega\right) \gg 1$ ) to lowest order $g\left(\psi_{*}\right)=0$ and (39) reduces to the relation

$$
a(\omega)=-a_{*}
$$

(46) has two roots

$$
\omega_{1,2}=\left[-a_{*} \omega_{*_{i}} \pm \sqrt{a_{*}^{2} \omega_{* i}^{2}-\left(1+2 a_{*}\right) \Gamma^{\prime 2}}\right] /\left(1+2 a_{*}\right)
$$

where both are real provided that

$$
\Gamma^{2}<\frac{a_{*}^{2}}{1+2 a_{*}} \omega_{*_{i}}^{2}
$$

Hence, the case $q / \psi_{*} \gg 1$ with (48) satisfied will be referred as the "stabilized" mode of operation. When it does not apply, then FLR effects do not stabilize the $m=1$ mode.

The "stabilized" mode of operation is in fact unstable with respect to a low frequency flute perturbation when dissipative effects are included. To prove this, we first note that the frequency $\omega$ is real at marginal stability while the alternative quadratic form

$$
\begin{aligned}
\mathcal{L}_{\mathrm{cl}}= & \frac{\pi e_{i}^{2} \phi_{0}^{2} l}{m_{i} \Omega_{i}^{2}} \int_{0}^{\infty} d \psi \times \\
& \left\{-\left[|g|^{2}+\left|g+2 \psi g^{\prime}\right|^{2}\right] n_{i}+4 \psi^{2}\left|g^{\prime}\right|^{2} n_{i}^{\prime} \frac{\omega_{*_{i}}}{\omega}+2 \psi|g|^{2} n_{i}^{\prime} \frac{\Gamma^{2}}{\omega^{2}}-2 i \psi|g|^{2} n_{i} \frac{\nu}{\omega}\right\}
\end{aligned}
$$

is equal to zero for the correct eigenfunction $g(\psi)$, i.e. $\mathcal{L}_{\mathrm{cl}}=0$. Next, we multiply the equation $\mathcal{L}_{\mathrm{cl}}=0$ by $\omega^{2}$ and separate the imaginary and real parts with $\omega$ being the real marginal frequency. The imaginary part of the equation yields that $\omega=0$. Substituting $\omega=0$ into the real part reveals that $\Gamma^{2}=0$ at the marginal stability frequency. We will 
show below that for all modes $\operatorname{Im} \omega<0$ only when $\Gamma^{2}<0$. Thus, the plasma is stable only if

$$
\Gamma^{2}<0
$$

while instability exists when $\Gamma^{2}>0$. However the instability is slow provided that the criterion (48) holds; instability formally disappears as $\nu \Delta \psi \rightarrow \infty$.

More detailed analysis reveals that the dispersion relation given by (39) is a cubic algebraic equation:

$$
\left(\omega+\omega_{*_{i}}+i \nu_{*}\right)\left(\omega^{2}+\Gamma^{2}\right)+2 i a_{*} \nu_{*}\left(\omega+\omega_{*_{i}}\right) \omega=0
$$

Therefore it has three roots.

For small $\nu$, the dissipation hardly changes the two MHD roots $\omega_{1}=i \Gamma$ and $\omega_{2}=-i \Gamma$. The third root is near $\omega_{*_{i}}$ and damped, $\omega_{3}=-\omega_{*_{i}}-i \nu_{*}$.

In the limit $\nu \rightarrow \infty$, we find that the MHD roots become

$$
\begin{aligned}
\omega_{1,2} & =\omega_{1,2}^{(0)}+i \operatorname{Im} \omega_{1,2} \\
\omega_{1,2}^{(0)} & =\frac{-a_{*} \omega_{*_{i}} \pm\left[a_{*}^{2} \omega_{*_{i}}^{2}-\left(1+2 a_{*}\right) \Gamma^{2}\right]^{1 / 2}}{1+2 a_{*}} \\
\operatorname{Im} \omega_{1,2} & =\mp \frac{\left(\omega_{1,2}^{(0)}+\omega_{*_{i}}\right)^{2} \omega_{1,2}^{(0)} a_{*}}{\nu_{*}\left[a_{*}^{2} \omega_{*_{i}}^{2}-\left(1+2 a_{*}\right) \Gamma^{2}\right]^{1 / 2}} \\
& =\mp \frac{\left[\left(\omega_{1,2}^{(0)}\right)^{2}+\Gamma^{2}\right]^{2}}{4 \nu_{*} a_{*} \omega_{1,2}^{(0)}\left[a_{*}^{2} \omega_{* i}^{2}-\left(1+2 a_{*}\right) \Gamma^{2}\right]^{1 / 2}} .
\end{aligned}
$$

Recall that in these expressions $a_{*} \ll 1$, which is necessary for the asymptotic approximation of ${ }_{1} F_{1}\left(a_{1} 2 ; \psi_{*}\right)$ to be accurate. The $\omega_{2}$ root is always stable (as $\omega_{2}^{(0)}$ is negative) independent of the sign of $\Gamma^{2}$, while the $\omega_{1}$ root is stable for $\Gamma^{2}<0$ (as then $\omega_{1}^{(0)}$ is positive) and unstable for $\Gamma^{2}>0$ (as then $\omega_{1}^{(0)}$ is negative). Thus the condition $\Gamma^{2}=0$ is marginal for the $\omega_{1}$ root. Near the marginal region we have

$$
\omega_{1} \simeq-\frac{\Gamma^{2}}{2 \omega_{*_{i}} a_{*}}+i \frac{\Gamma^{2}}{2 \nu_{*} a_{*}}
$$


A third root

$$
\omega_{3} \simeq-i\left(1+2 a_{*}\right) \nu_{*}
$$

is strongly damped and uninteresting.

Let us apply the technique described above to find the marginal condition for (51). Assuming $\omega$ to be real and separating (51) into the real and imaginary parts, we obtain two equations. Solving them together reveals that, in addition to $\Gamma^{2}=0$, there is one more point, $\Gamma^{2}=-\omega_{*_{i}}^{2}$, where the frequency $\omega_{2}=-\omega_{*_{i}}$ is purely real. However, the latter point is not marginal. From (52) we find that the imaginary purt of the eigenfrequency

$$
\omega_{2} \simeq-\omega_{*_{i}}+\frac{\Gamma^{2}+\omega_{*_{i}}^{2}}{2\left(1+a_{*}\right) \omega_{*_{i}}}-i \frac{\left(\Gamma^{2}+\omega_{*_{i}}^{2}\right)^{2} a_{*}}{4\left(1+a_{*}\right)^{3} \omega_{*_{i}}^{2} \nu_{*}}
$$

does not change its sign at the point. We shall see in the next section that the point $\Gamma^{2}=-\omega_{*_{i}}^{2}$ lies on a "double marginal line" which split into two true marginal curves when dissipation due to energy spread of hot particles is introduced.

The existence of one marginal condition for three roots means, that two of three eigen modes never change their stability properties. We saw that in the case under consideration these two modes, namely the $\omega_{2}$ and $\omega_{3}$ modes, are always stable. The $\omega_{1}$ mode is unstable if $\left(-\Gamma^{2}\right)<0$. However the growth rate of this mode decreases as $\nu_{*} \rightarrow \infty$ if $\Gamma^{2}>a_{*}^{2} \omega_{*_{i}}^{2} /(1+$ $\left.2 a_{*}\right)$. Figure 2 shows $\operatorname{Im} \omega_{1}$ versus $\nu_{*}$ for various $\omega_{*_{i}}$.

Combining (34), (38), and (39) readily gives a value of $g$ at the limiter's edge:

$$
g\left(\dot{\psi}_{*}\right)=\left[1+\frac{i \nu_{*}}{\omega+\omega_{*_{i}}}\right]^{-1} .
$$

Since $g(0)=1$ at the plasma's axis, $g\left(\psi_{*}\right)$ gives insight into of the mode's radial structure. When plasma is detached from the limiter, i.e. $\nu_{*} /\left(\omega+\omega_{*_{i}}\right) \ll 1$, two MHD modes (with the frequencies $\omega_{1}$ and $\left.\omega_{2}\right)$ are rigid because $g\left(\psi_{\star}\right) \simeq 1$ while the third, i.e. the fast damping mode is located near the limiter's edge since $g\left(\psi_{*}\right) \rightarrow \infty$. In the opposite case, $\nu_{*} /\left(\omega+\omega_{*_{i}}\right) \gg 1$, the MHD modes tend to zero since $g\left(\psi_{*}\right) \simeq-i\left(\omega_{*_{i}}+\omega\right) / \nu_{*}$; on the contrary, the third mode once again is localized near the limiter as $g\left(\psi_{*}\right) \simeq\left(1+2 a_{*}\right) / 2 a_{*} \gg 1$. 
For the purpose of comparison of the theory with an experimental data, it is useful to specify the state of the system by the resistance $R=\phi / \int_{r_{*}}^{\infty} d r 2 \pi r\left(\delta j_{\| i}+\delta j_{\| e}\right)$ between the plasma and the limiter. As we saw, a plasma can be considered as detached from limiter (and from any surrounding conducting elements of an experimental device) if $\nu_{*} \ll \omega_{*_{i}}$; in terms of $R$, this condition takes the form.

$$
R>R_{*} \sim \frac{V_{\mathrm{A} *}^{2} / c^{2}}{l \omega_{*_{i}} \psi_{*}}
$$

where $V_{\mathrm{A} *}=B / \sqrt{4 \pi m_{i} n_{* i}}$ stands for the local value of the Alfvén velocity near the conductors.

The remaining part of the section we devote to the calculation of the radial structure of the eigenmodes in the special case of linear density drop at the limiter edge. Let

$$
n_{i}= \begin{cases}n_{0 i} \exp (-\psi), & \text { if } \quad \psi<\psi_{*} \\ n_{0 i} \exp \left(-\psi_{*}\right)\left[1-\left(\psi-\psi_{*}\right) / \Delta \psi_{*}\right], & \text { if } \quad \psi_{*}<\psi<\psi_{*}+\Delta \psi_{*} \\ 0, & \text { if } \quad \psi_{*}+\Delta \psi_{*}<\psi\end{cases}
$$

with the density gradient in the scrape-off layer being large, i.e. $\Delta \psi_{*} \ll 1$. The function $g\left(\psi^{\prime}\right)$ is once again expressed in terms of Kummer's function (34) in the region $\psi<\psi_{*}$, not shadowed by the limiter. When solving (30) in the sharp gradient region $\psi_{*}<\psi<\psi_{*}+\Delta \psi_{*}$, we take into account that $\Delta \psi \ll 1$ to reduce the equation to the form

$$
\frac{\omega_{*_{i}}}{\omega} \psi_{*} g^{\prime \prime}+\left(2 \frac{\omega_{*_{i}}}{\omega}-\psi_{*}\right) g^{\prime}-\frac{1}{2}\left(1+\frac{\Gamma^{2}}{\omega^{2}}\right) g+i \frac{\nu}{2 \omega}\left[\psi-\psi_{*}-\Delta \psi_{*}\right] g=0 \text {. }
$$

It.s general solution can be expressed in terms of the Bessel's functions $\mathrm{J}_{ \pm 1 / 4}$. However, it is more informative to seek a solution that is a power series in $x=\psi-\psi_{*}-\Delta \psi_{*}$. According to $(32) g^{\prime}=0$ at $x=0$ and $g=g\left(\psi_{*}\right)$ at $x=-\Delta \psi_{*}$. Hence, we have

$$
g=\left[1+\frac{1+\Gamma^{2} / \omega^{2}}{2 \psi_{*} \omega_{*_{i}} / \omega} \frac{x^{2}-\Delta \psi_{*}^{2}}{2}-\frac{i \nu / 2 \omega}{2 \psi_{*} \omega_{*_{i}} / \omega} \frac{x^{3}+\Delta \psi_{*}^{3}}{3}\right] g\left(\psi_{*}\right)
$$

Providing the condition $(40)$ holds, the neglected terms are $\Delta \psi_{*} / \psi_{*}$ times smaller as compared with those kept in (55). For the same condition, the first term in the brackets, i.e. 
1 , is greater than the others. These conditions then imply, as previously noted, that $g$ is constant in the scrape-off layer.

Matching derivatives of (55) and (34) at $\psi=\psi_{*}$ by means of (31) reproduces the dispersion relation (39) if we note that $\Delta \psi=\Delta \psi_{*} / 2$ in agreement with (33).

The radial profile of the perturbation $g=\left(\widehat{\phi} / \phi_{0}\right) /\left(r / r_{0}\right)$ is almost flat if $\nu_{*} /\left(\omega+\omega_{*_{i}}\right) \ll 1$. This is the rigid mode. In the opposite case, $\nu_{*} /\left(\omega+\omega_{*_{i}}\right) \gg 1$, the perturbation tends to zero at the limiter's edge. This is the "stabilized" mode. In the intermediate case, $\nu_{*} /\left(\omega+\omega_{*_{i}}\right) \sim 1, g$ changes appreciably near the plasma's side of the limiter's edge but it is almost flat in the shadow of the limiter.

\section{Two-Component Plasma}

In this section we consıder MHD stability of plasma consisting of a relatively cold core (background plasma) and a high er argy hot ions species. We assume that the hot ion density is much smaller than that of the plasma core density, $n_{h} \ll n_{c}$, while their pressures have the inverse relati: $\mathrm{n}$, so that $n_{h} / n_{c} \gg\left(\rho_{i} / \rho_{h}\right)^{2}$. The quadratic form, relevant to the situation, is the si'm of (22), (25), and (28), i.e.

$$
L_{\mathrm{chl}}=L_{\mathrm{c}}+L_{\mathrm{h}}+L_{\mathrm{l}}
$$

Let the distribution of the hot ions be quasimonoenergetic, i.e. the velocity spread $\Delta v$ is much smaller than the average velocity $v_{0}, \Delta v \ll v_{0}$. Such a situation occurs when hot ions escape the plasma in the result of charge exchange processes. As an approximation we neglect the angular spread because typically in two-component plasma the spread is small as electron drag causes hot ions to slow down much faster than they scatter. Thus, the distribution of hot ions, peaked near the pitch angle $\vartheta_{L}=\vartheta_{0}$, where $\cos \theta_{L}=v_{\|} / v_{L}$, can be modelled by the following function

$$
F_{h}\left(\mathbf{r}_{G}, \mathbf{v}_{L}\right)=\frac{n_{h}\left(r_{G}\right) \Delta v}{4 \pi^{2} v_{0}^{2}} \sum_{ \pm} \frac{\delta\left(\cos \vartheta_{L} \pm \cos \vartheta_{0}\right) h\left(v_{L}\right)}{\left(v_{L}-v_{0}\right)^{2}+(\Delta v)^{2}}
$$


where $h\left(v_{0}\right)=1$ and $h\left(v_{L}\right)$ varies on a scale $v_{L} \sim v_{0}$ while $\Delta v / v_{0} \ll 1$. Using (56), allows us to perform the integration over velocity in (25). In the integration, we can then neglect the dependence of the Larmor radius $r_{L}=\left(v_{L} / \Omega_{h}\right) \sin \vartheta_{L}$ on the velocity spread though we take into account that $\omega_{\kappa h}$ is proportional to $v_{L}^{2}$. Then we find

$$
\begin{aligned}
L_{\mathrm{h}}= & \frac{2 e_{h}^{2}}{m_{h} \Omega_{h}^{2}} \int d^{3} r_{G} \times \\
& {\left[\frac{\Omega_{h}}{\omega}\left\langle\frac{\hat{\phi}^{2}}{r^{2}}\left(r_{G}^{2}+r_{G} \rho_{h} \cos \chi\right)\right\rangle-\frac{\Omega_{h}}{\omega-\omega_{\kappa h}+i \gamma}\left\langle\frac{\widehat{\phi}}{r}\left(r_{G}+\rho_{h} \cos \chi\right)\right\rangle^{2}\right] \frac{\partial n_{h}}{\partial r_{G}^{2}} . }
\end{aligned}
$$

where (and in what follows) $\omega_{\kappa h}$ stands for the value of hot ions drift frequency at $v_{L}=v_{0}$, $\rho_{h}=\left(v_{0} / \Omega_{h}\right) \sin \vartheta_{0}, \gamma$ is a positive function of the ratio $\omega / \omega_{\kappa h}, \gamma=\gamma\left(\omega / \omega_{\kappa h}\right) \leq 0$. For purely real frequency $\omega$ we have $\gamma=2\left(\Delta v / v_{0}\right)\left|\omega_{\kappa h}\right|$ if $\omega / \omega_{\kappa h}>0$ while $\gamma=0$ if $\omega / \omega_{\kappa h}<0$ since then there are no resonant particles, hence no dissipation. Our model is accurate if $\left|\left(\omega-\omega_{\kappa h}\right) / \omega_{\kappa h}\right| \ll 1$, while if $\left|\left(\omega-\omega_{\kappa h}\right) / \omega_{\kappa h}\right| \sim 1$, our model only has qualitative significance. For the sake of simplicity, we assume, just in this section, that $\rho_{h} \ll r_{0}$ and apply the expansion procedure, described in $\S 3$., to (57). It yields

$$
L_{\mathrm{h}}=\frac{2 \pi e_{h}^{2} l \phi_{0}^{2}}{m_{h} \Omega_{h}^{2}} \int d \psi\left[-\frac{\Omega_{h}\left(\omega_{\kappa h}-i \gamma\right)}{\omega\left(\omega-\omega_{\kappa h}+i \gamma\right)} \psi g^{2}+2 \frac{\omega_{* h}}{\omega} \psi^{2} g^{\prime 2}\right] n_{h}^{\prime}
$$

Suppose also that the relative density profiles of the hot ions $n_{h}(\psi)$ replicates that of core plasma, i.e. $n_{h}(\psi) / n_{i}(\psi)=$ const. It allows us to reuse the results of previous section. In particular, the dispersion relation (39) remains valid after redefining the notations $a(\omega)$ and $q(\omega)$ as follows:

$$
\begin{gathered}
a(\omega) \equiv \frac{1}{2} \frac{1+\Gamma^{2} / \omega^{2}-\left(\omega_{\kappa h}-i \gamma\right) \Omega_{h} \mu / \omega\left(\omega-\omega_{\kappa h}+i \gamma\right)}{1+\left(\omega_{*_{i}}+\omega_{*_{h}} \mu\right) / \omega} \\
q(\omega) \equiv \frac{1}{2} \frac{\nu \Delta \psi / \omega}{1+\left(\omega_{*_{i}}+\omega_{*_{h}} \mu\right) / \omega}
\end{gathered}
$$

where $\mu=m_{h} n_{0 h} / m_{i} n_{0 i}$ is the ratio of the total mass of hot ion species to that of background ions. Thus we get the following dispersion relation

$$
\left[\left(\omega^{2}+\Gamma^{2}\right)\left(\omega-\omega_{\kappa h}+i \gamma\right)-\left(\omega_{\kappa h}-i \gamma\right) \Omega_{h} \omega \mu\right]\left[1-i\left(\omega+\omega_{*_{i}}+\omega_{* h} \mu\right) / \nu_{*}\right]
$$




$$
+2 a_{*}\left(\omega+\omega_{* i}+\omega_{* h} \mu\right)\left(\omega-\omega_{\kappa h}+i \gamma\right) \omega=0
$$

\subsection{Effect of escaping particles}

We begin to analyze the dispersion relation (61) with the case of monoenergetic hot ions, so that $\gamma=0$.

\subsubsection{Rigid mode}

Let $\gamma=0$ and $\nu_{*}=0$. Then (61) becomes the cubic equation with real coefficients:

$$
\left(\nu^{2}+\Gamma^{2}\right)\left(\omega-\omega_{\kappa h}\right)-\omega_{\kappa h} \Omega_{h} \omega \mu=0 .
$$

If $\left|\omega_{\kappa h} \Omega_{h}\right| \mu \ll\left|\Gamma^{2}\right|$, the hot ion modification doesn't alter the two MHD modes (45), which exist in one-component plasma, and give rise to a new stable mode (the precessional mode) with the frequency $\omega_{3} \simeq \omega_{\kappa h}$. In the opposite limit, $\left|\omega_{\kappa h} \Omega_{h}\right| \mu \gg \Gamma^{2}$ it is the hot ions that determine stability properties of the plasma. The roots are: $\omega_{1} \simeq-\Gamma^{2} / \Omega_{h} \mu, \omega_{2,3} \simeq$ $\pm \sqrt{\omega_{\kappa h} \Omega_{h} \mu}$.

Marginal stability corresponds tu the case when two of the three roots are equal. This means that at marginal stability the derivative of (62) with respect to $\omega$ is also equal to zero:

$$
\left(\omega^{2}+\Gamma^{2}\right)+2 \omega\left(\omega-\omega_{\kappa h}\right)-\omega_{\kappa h} \Omega_{h} \mu=0 .
$$

Solving (62) and (63) together, we get the parametric expression for marginal stability that has been found earliei by Mikhaylovskaya (1984):

$$
\Gamma^{\prime} / \omega_{\kappa h}^{2}=-(2 f-1) f^{2}, \quad\left(\Omega_{h} / \omega_{\kappa h}\right) \mu=-2(f-1)^{2} f
$$

where $f=\omega / \omega_{\kappa h}$ is the real parameter which takes on values such that $\mu \geq 0$, nameiy, $f>0$ if $\omega_{\kappa h}<0$ and $f<0$ if $\omega_{\kappa h}>0$. This marginal condition is drawn in figure 3; it can be called the MHD marginal stability condition because it is found without taking into account the dissipative processes described by the frequencies $\gamma$ and $\nu$. 
In this diagram the upper right corresponds tc both the hot particles and background particles sampling good curvature, and this region is always stable.

In the upper left the hot particles sample good curvature, and the background plasma bad curvature. A simple formula for stability can be given if $\Gamma^{2} / \omega_{\kappa h}^{2}$ is either much less or much larger then unity. If $\Gamma^{2} / \omega_{\kappa h}^{2} \ll 1$, the stability requires $\Omega_{h} \mu / \omega_{\kappa h}>2 \Gamma^{2} / \omega_{\kappa h}^{2}$ while if $\Gamma^{2} / \omega_{\kappa h}^{2} \gg 1$ stability requ'.es $\Omega_{h} \mu / \omega_{\kappa h}>\Gamma^{2} / \omega_{\kappa h}^{2}$. An interpolation formula between the two regions of $\Gamma$ gives as stability condition $\Omega_{h} \mu / \omega_{\kappa h}>\Gamma^{2} / \omega_{\kappa h}^{2}+2 \Gamma / \omega_{\kappa h}$.

The lower right corresponds to the hot particles sampling bad curvature and the background particles good curvature. When the background pressure is large enough one of course has stability. However, even for low background pressure there is a stability region. This is due to the stability mechanism first discussed by Krall (1966), who observed that for hot particles in a cold background plasma (where $\Gamma^{2}$ is small) stability arises if $\mu$ is sufficiently small. Finally, the lower left region corresponds to both the hot particles and background particles sampling bad curvature. If $\mu$ is sufficiently small, the instability is only affecting the background plasma, and hot particles should remain relatively well contained. The small stability region in this quadrant is due to a charge uncovering effect. The perturbed $\mathbf{E} \times \mathbf{B}$ drift of background electrons and ions produce a radial current, hence a charge separation. since their densities are not equal, as the hot particles do not respond to the low frequency perturbation if $\omega \ll \omega_{\kappa h}$. This charge separation produces stability if (here $\left.\Gamma^{2}>0, \omega_{\kappa h} / \Omega_{h}<0\right)$

$$
-\frac{\omega_{\kappa h}}{4 \Omega_{h}} \gtrsim \mu \gtrsim \frac{2 \Gamma}{\Omega_{h}} .
$$

The right-hand side of the inequality is needed to achieve enough charge separation to stabilize, while the left-hand side of the inequality is needed to prevent the hot particle curvature drive from exciting instability. The stabilization due to charge uncovering has been studied previously (e.g. (Berk, Rosenbluth, Van Dam, Spong 1983; and Timofeev 1979)). 


\subsubsection{Stabilized mode}

For $\gamma=0$ and $\nu_{*}=\infty$ the dispersion relation (61) is again a cubic equation with real coefficients:

$$
\left(\omega^{2}+\Gamma^{2}\right)\left(\omega-\omega_{\kappa h}\right)-\omega_{\kappa h} \Omega_{h} \omega \mu+2 a_{*}\left(\omega+\omega_{* ;}+\omega_{* h} \mu\right)\left(\omega-\omega_{\kappa h}\right) \omega=0 .
$$

Using the same method as before, we obtain the parametric expression for the MHD marginal stability condition:

$$
\begin{aligned}
& \Gamma^{2} / \omega_{\kappa h}^{2}=-\frac{\left\{\left(1+2 a_{*}\right)\left[f^{2}-\left(1+2 a_{*} \omega_{* h} / \Omega_{h}\right)(f-1)^{2}\right]+2 a_{*} \omega_{* i} / \omega_{\kappa h}\right\} f^{2}}{1+2 a_{*}(f-1)^{2} \omega_{* h} / \Omega_{h}}, \\
& \left(\Omega_{h} / \omega_{\kappa h}\right) \mu=-2 \frac{\left[\left(1+2 a_{*}\right) f+a_{*} \omega_{*_{i}} / \omega_{\kappa h}\right](f-1)^{2}}{1+2 a_{*}(f-1)^{2} \omega_{* h} / \Omega_{h}}
\end{aligned}
$$

it is shown on figure 4 for $\omega_{*_{i}} / \mid \omega_{\kappa h}=0$ and on figure 6 for $\omega_{*_{i}} / \mid \omega_{\kappa h}=1$. In figures 5 and 7 the contents of figure 4 and 6 is presented on a finer scale. Here $f=\omega / \omega_{\kappa h}$ is the real parameter such that $\mu>0$. More precisely, if $\omega_{\kappa h}>0, f<-\left[a_{*} /\left(1+2 a_{*}\right)\right]\left(\omega_{*_{i}} / \omega_{\kappa h}\right)<0$, and consequently $\gamma=0$, while if $\omega_{\kappa h}<0$, then $\gamma \neq 0$, as $f>-\left[a_{*} /\left(1+2 a_{*}\right)\right]\left(\omega_{\star_{i}} / \omega_{\kappa h}\right)>0$. We will make use of such behavior of $\gamma$ along the MHD marginal curves in the next section.

These curves show that stability improves with finite $a_{*}$ (i.e. as the radius $r_{*}$ of the limiter decreases). The curves also indicate that when $\omega_{\kappa h}>0$ and $-\Gamma^{2}<0$ there is an appreciable band of instability as hot particles do not respond to the MHD motion. The mode is then unstatile to the MHD mode involving only background plasma dynamics. To obtain stabilization from the good curvature of the hot particles, the hot particle curvature drift has to reduce to a value less than the frequency $\sim \Gamma$ of the background plasma. Hence, only when $\omega_{\kappa h}<\Gamma$ can the hot particles respond in an MHD manner. Then if $\mu \omega_{\kappa h}>\left|\omega_{\kappa i}-\omega_{\kappa e}\right|$, will stability of the MHD mode be effected. It appears that finite Larmor radius effects broaden the stability region.

When $\omega_{\kappa h}<0$ (lower half of figures 5 and 7 ) we see that for $\left(-\Gamma^{2} / \omega_{\kappa h}^{2}\right) \sim 1$ and $\mu \Omega_{h} /\left|\omega_{\kappa h}\right| \sim 1$ the marginal instability is insensitive to the hot particle Larmor radius $\rho_{h}$. 
However, as $\mu \Omega_{h} /\left|\omega_{\kappa h}\right|$ increases dependence of marginal stability on $p_{h}$ arises. The return of curve one to the lower left quadrant arises because hot ion FLR effects can stabilize only when $\left|\omega_{\kappa h}\right|<\Gamma$. Then the following FLR stabilization condition applies

$$
\left[a_{*}\left(\omega_{*_{i}}+\mu \omega_{* h}\right)\right]^{2}>\Gamma^{2}-\omega_{\kappa h} \Omega_{h} \mu
$$

\subsubsection{Intermediate case}

Let now $\nu_{*}$ be finite while still $\gamma=0$. As was explained in the $\S 4$., the region of stability is then substantially smaller than ccmpared with the MHD stable region determined by (64) and (66) when $\nu_{*}=0$ and $\nu_{*}=\infty$ respectively. However, the instability, which appears due to dissipation (i.e. due to leakage of the background plasma onto the limiter) inside the region of MHD stability is weak provided that $\nu_{*} \rightarrow 0$ or $\nu_{*} \rightarrow \infty$.

To find the marginal stability condition, we use the technique described in $\S 4$.. Let us consider the equation $\omega^{2}\left(\omega-\omega_{\kappa h}\right) \mathcal{L}_{\text {chl }}=0$ at marginal stability. Hence the frequency is real. As the dependence on the field amplitudes occur in complex conjugate pairs, the complex nature of this expression only arises from the explicit dissipative terms. The imaginary part of the equation yields that $\omega=0$ or $\omega=\omega_{\kappa h}$. Consequently substituting $\omega=0$ and $\omega=\omega_{\kappa h}$ into the real part of the equation yields respectively $\Gamma^{2}=0$ and $\omega_{\kappa h}=0$. These two lines divide the plane $\left\{\Gamma^{2} / \omega_{\kappa h}^{2},\left(\Omega_{h} / \omega_{\kappa h}\right) \mu\right\}$, shown in figures $3-7$, into four quadrants. As we shall show, only one of them, namely

$$
-\Gamma^{2}>0, \quad \omega_{\kappa h} \Omega_{h}>0 .
$$

corresponds to the case when all modes are stable. This is compatible with the result originally obtained by Greene and Coppi (1965).

The line $\Gamma^{2}=0$ is marginal for the low frequency mode, $\omega=\omega_{1}$, such that $\omega \rightarrow 0$ as $\Gamma^{2} \rightarrow 0$. This mode is stable when $\Gamma^{2}<0$ since with sufficiently small $\Gamma^{2}$,

$$
\operatorname{Im} \omega_{1}=\frac{2 a_{*}\left(\omega_{*_{i}}+\omega_{* h} \mu\right)^{2} / \nu_{*}}{\left[\Omega_{h} \mu+2 a_{*}\left(\omega_{*_{i}}+\omega_{* h} \mu\right)\right]^{2}+\left[\left(\omega_{*_{i}}+\omega_{*_{h}} \mu\right) \Omega_{h} \mu / \nu_{*}\right]^{2}} \Gamma^{2} .
$$


Another line $\omega_{\kappa h}=0$ is marginal for the "precessional" mode which has the property that as $\mu \rightarrow 0, \omega=\omega_{3} \rightarrow \omega_{\kappa h}$. This mode is stable when $\left(\Omega_{h} / \omega_{\kappa h}\right) \mu>0$ since for sufficiently small $\mu$

$$
\operatorname{Im} \omega_{3}=-\frac{2 a_{*} \omega_{\kappa h}^{4}\left(\omega_{* i}+\omega_{\kappa h}\right)^{2} / \nu_{*}}{\left[\omega_{\kappa h}^{2}+\Gamma^{2}+2 a_{*}\left(\omega_{*_{i}}+\omega_{\kappa h}\right) \omega_{\kappa h}\right]^{2}+\left[\left(\omega_{\kappa h}^{2}+\Gamma^{2}\right)\left(\omega_{* i}+\omega_{\kappa h}\right) / \nu_{*}\right]^{2}} \frac{\Omega_{h} \mu}{\omega_{\kappa h}} .
$$

Repeating the reasoning of $\S 4$., we come to the conclusion that the instability, existing in-between the MHD marginal condition given by (66), and the absolute marginal condition given by $(67)$, is weak when $\nu_{*} /\left(\omega+\omega_{*_{i}}\right) \gg 1$; it disappears as $\nu_{*} /\left(\omega+\omega_{*_{i}}\right) \rightarrow \infty$. Similarly, the growth rates go to zero in the region between the marginal condition given by (64) and $(67)$ if $\nu_{*} /\left(\omega+\omega_{* i}\right) \rightarrow 0$.

These instabilities can be interpreted as follows. The outflow of the plasma produces positive dissipation. The FLR effect from having a finite $a_{*}$, produces a positive energy wave ( $\omega_{2}$ mode) and negative energy wave $\left(\omega_{1}\right.$ mode) if $\left(-\Gamma^{2}\right)<0$. This negative energy wave is destabilized by positive dissipation. Similarly, the precessional mode, if $\omega_{\kappa h}<0$, is also a negative energy wave, that is destabilized by positive dissipation. When $\left(-\Gamma^{2}\right)>0$, the low frequency negative energy wave (the $\omega_{1}$ mode) converts to a positive energy wave, which is then stable to the dissipation. Similarly, the precessional frequency mode converts to positive energy when $\omega_{\kappa h}>0$, which damps due to positive dissipation.

The dispersion relation (61) is an algebraic equation of fourth power. Therefore it has four roots while the equation $L_{\mathrm{chl}}=0$ for a given perturbation profile $g(\psi)$ (when assumed independent of $\omega$ ) has only three roots. We found in $\S 4$. that a similar situation takes place for a one-component plasma, i.e. the dispersion equation had one more root as compared to the number of apparent roots of the equation $\mathcal{L}_{\mathrm{cl}}=0$ taken with a test function that is formally independent of $\omega$. It was also found that the additional mode is peaked near the limiter's edge while other modes have similar radial profiles that are peaked near the plasma's axis. Therefore it is not surprising that when a test function is taken that is assumed 
spatially broad, that one does not recover a mode from a variational form that is localized near the edge.

Searching for a real roots of the dispersion relation (61) gives one more candidate for marginal stability in addition to two marginal modes found above:

$$
\mu=-\frac{\omega+\omega_{*_{i}}}{\omega_{* h}}, \quad \Gamma^{2}=-\omega^{2}-\frac{\left(\omega+\omega_{*_{i}}\right) \omega \Omega_{h} \omega_{\kappa h}}{\left(\omega-\omega_{\kappa h}\right) \omega_{* h}}
$$

where $\omega<-\omega_{*_{i}}$ is real parameter. However this line is not marginal, because the eigenfrequency, which is real on the line, have an imaginary part of the same sign on both sides of the line. Generally speaking, the sign of its imaginary sign reverses at several points, where two of the four modes have one and the same real frequency. Hence, the existence of such points inside any of the quadrants would indicate existence of an unstable eigenmode. One can show that there are no such points in the quadrant defined by (67), where all four modes are stable. We shall see that (68) splits for two marginal modes when finite energy spread of the hot ions are being taken into account; therefore this line can be called a double marginal line.

\subsection{Effect of energy spread}

We now proceed to the effect of hot ion energy spread on the stability. To take this effect into account, we allow for the parameter $\gamma$ to be finite, $\gamma \neq 0$. This introduces negative dissipation when $\omega_{\kappa h}<0$, which can destabilize positive energy waves such as $\omega_{3}$. However, when both positive and negative dissipation are present, the region of stability can be expanded compared with the cases where one of the dissipaticn mechanisms are ignored.

\subsubsection{Stabilized mode}

First, we assume, that $\nu_{*}=\infty$. In this case, the dispersion relation (61) becomes a cubic equation with complex coefficients:

$$
\left(\omega^{2}+\Gamma^{2}\right)\left(\omega-\omega_{\kappa h}+i \gamma\right)-\left(\omega_{\kappa h}-i \gamma\right) \Omega_{h} \omega \mu+2 a_{\star}\left(\omega+\omega_{* i}+\omega_{\star h} \mu\right)\left(\omega-\omega_{\kappa h}+i \gamma\right) \omega=0
$$


The solution for $\omega$ has a real frequency if $(i) \Gamma^{2}=0$ or $(i i) \mu=0, \Gamma^{2} \leq a_{*}^{2} \omega_{*_{i}}^{2} /\left(1+2 a_{*}\right)$.

(i) If $\Gamma^{2} \rightarrow 0$ and $\mu$ is arbitrary, the real eigenfrequency is $\omega=0$. Taking into account finite $\Gamma^{2}$ gives

$$
\omega_{1}=-\frac{\Gamma^{2}}{\Omega_{h} \mu+2 a_{*}\left(\omega_{*_{i}}+\omega_{*_{h}} \mu\right)}-\frac{i \gamma \Omega_{h} \mu \Gamma^{4}}{\left[\Omega_{h} \mu+2 a_{*}\left(\omega_{*_{i}}+\omega \omega_{h} \mu\right)\right]^{3} \omega_{\kappa h}^{2}},
$$

where recall $\gamma=0$ for $\omega / \omega_{\kappa h}<0$. Thus if $\omega_{\kappa h}<0, \omega_{1}$ is damped if $\left(-\Gamma^{2}\right)^{\circ}<0$ and purely oscillatory if $\left(-\Gamma^{2}\right)>0$. If $\omega_{\kappa h}>0$ the mode is damped if $\left(-\Gamma^{2}\right)>0$ and purely oscillatory if $\left(-\Gamma^{2}\right)<0$.

(ii) If $\mu=0, \Gamma^{2} \leq a_{*}^{2} \omega_{\boldsymbol{*}_{i}}^{2} /\left(1+2 a_{\boldsymbol{*}}\right)$, there are two real eigenfrequencies $\omega_{1,2}^{(0)}$ which are given by (47). When $\mu$ is finite (i.e. $\mu>0$ ) but small,

$$
\begin{aligned}
& \omega_{1,2} \approx \omega_{1,2}^{(r)}+i \operatorname{Im} \omega_{1,2}, \\
& \omega_{1,2}^{(0)}=\frac{-a_{*} \omega_{* i} \pm\left[a_{*}^{2} \omega_{* i}^{2}-\left(1+2 a_{*} \Gamma^{2}\right)\right]^{1 / 2}}{1+2 a_{*}}, \\
& \operatorname{Im} \omega_{1,2}=\mp \frac{i \mu \Omega_{h} \omega_{1,2}^{(0)} \gamma}{2\left[\left(\omega_{1,2}^{(0)}-\omega_{\kappa h}\right)^{2}+\gamma^{2}\right]\left[a_{*}^{2} \omega_{*_{i}}^{2}-\left(1+2 a_{*}\right) \Gamma^{2}\right]^{1 / 2}},
\end{aligned}
$$

with the upper sign (lower sign) associated with $\omega_{1}\left(\omega_{2}\right)$. Recall $\gamma=0$ if $\omega / \omega_{\kappa h}<0$ and note that $\omega_{2}^{(0)}<0$ while $\omega_{1}^{(0)}$, which is also negative for $\left(-\Gamma^{2}\right)<0$, changes its sign for $\left(-\Gamma^{2}\right)>0$.

These results can be interpreted as follows:

(a) If $\left(-a_{*}^{2} \omega_{*_{i}}^{2}\right) /\left(1+2 a_{*}\right)<\left(-\Gamma^{2}\right)<0$ and $\omega_{\kappa h}<0$, then the negative dissipation arising from the hot particle spread destabilizes the positive energy $\omega_{2}$ mode and stabilizes the negative energy $\omega_{1}$ mode.

(b) If $\left(-a_{*}^{2} \omega_{\boldsymbol{*}_{i}}^{2}\right) /\left(1+2 a_{*}\right)<\left(-\Gamma^{2}\right)<0$ and $\omega_{\kappa h}>0$, the $\omega_{2}$ mode and $\omega_{1}$ mode, both have the property that $\omega / \omega_{\kappa h}<0$, so that $\gamma=0$ and the modes are purely oscillatory.

(c) If $\left(-\Gamma^{2}\right)>0$ and $\omega_{\kappa h}>0$, the dissipation from hot particles is positive, which damps the $\omega_{1}$ mode which is now a positive energy wave, while the $\omega_{2}$ wave is undamped as $\omega_{2} / \omega_{\kappa h}<0$. 
(d) If $\left(-\Gamma^{2}\right)>0$ and $\omega_{\kappa h}<0$ the $\omega_{2}$ mode is destabilized and the $\omega_{1}$ mode is undamped.

(e) If $\left(-\Gamma^{2}\right)<\left(-a_{*}^{2} \omega_{* i}^{2}\right) /\left(1+2 a_{*}\right)$ the $\omega_{1}$ mode is MHD unstable.

Finally, the third, precussional mode is approximately given by $\omega_{3}=\omega_{\kappa h}-i \gamma$, and is damped.

In order to extrapolate the above cited conclusions to the case where $\mu$ is not small, we first of all note that, except for the line interval defined by $\left\{\mu=0,\left(-\Gamma^{2}\right)>\left(-a_{*}^{2} \omega_{*_{i}}^{2}\right) /(1+\right.$ $\left.\left.2 a_{*}\right)\right\}$ (see figures 4-7) and the MHD marginal curves (66) there are no other marginal conditions. Taking into account the behavior of $\gamma$ along the MHD marginal curves (see $\S 5.1 .2$ ), we conclude that the region of absolute stability now extends to the right the MHD marginal curve in the upper half $\left(\omega_{\kappa h}>0\right)$ of figures $4-7$ and above the line $\Omega_{h} \mu / \omega_{\kappa h}=0$ for $\left(-\Gamma^{2}\right)>\left(-a_{*}^{2} \omega_{*_{i}}^{2}\right) /\left(1+2 a_{*}\right)$.

\subsubsection{Rigid mode}

Replacing $a_{*}$ by 0 in the formulas of the previous subsection, we have the case of the rigid mode. In the absence of dissipation this mode can be stabilized by finite $\mu$ in a small region where $\left(-\Gamma^{2}\right)<0, \omega_{\kappa h}<0$ (charge uncovering mechanism) or in the region $\left(-\Gamma^{2}\right)>0$, $\omega_{\kappa h}<0$ (the Krall decoupling mechanism). However, as before dissipation will destabilize the mode unless $\left(-\Gamma^{2}\right)>0$ and $\omega_{\kappa h}>0$. For $\omega_{1,2} \ll \omega_{\kappa h}$ the mode frequency is

$$
\begin{gathered}
\omega_{1,2} \approx \omega_{1,2}^{(0)}+i \operatorname{Im} \omega_{1,2} \\
\omega_{1,2}^{(0)}=-\mu \Omega_{h} / 2 \pm\left[\mu^{2} \Omega_{h}^{2} / 4-\Gamma^{2}\right]^{1 / 2} \\
\operatorname{Im} \omega_{1,2}=\mp \frac{i \gamma\left(\omega_{1,2}^{(0)}\right)^{2} \Omega_{h}}{2 \omega_{\kappa h}^{2}\left[\mu^{2} \Omega_{h}^{2} / 4-\Gamma^{2}\right]^{1 / 2}} .
\end{gathered}
$$

The structure of this formula is the same as (71), and similar conclusions apply; viz. (a) $\left(-\mu^{2} \Omega_{h}^{2} / 4\right)<\left(-\Gamma^{2}\right)<0, \omega_{\kappa h}<0$; the $\omega_{1}$ mode is damped, the $\omega_{2}$ mode is unstable,

(b) $\left(-\mu^{2} \Omega_{h}^{2} / 4\right)<\left(-\Gamma^{2}\right)<0, \omega_{\kappa h}>0$; the $\omega_{1}$ and $\omega_{2}$ modes are oscillatory, (c) $\left(-\Gamma^{2}\right)>0$, 
$\omega_{\kappa h}<0$; then the $\omega_{2}$ mode is unstable while the $\omega_{1}$ mode is purely oscillatory, $(\mathrm{d})\left(-\Gamma^{2}\right)>0$, $\omega_{\kappa h}<0$, then the $\omega_{2}$ mode is purely oscillatory, and the $\omega_{1}$ mode is damped.

Finally, the precessional mode, with $\omega \approx \omega_{\kappa h}-i \gamma$ is damped.

Stability is to the region right of the MHD marginal curve in the upper half $\left(\omega_{\kappa h}>0\right)$ of figures $4-7$.

\subsection{Combined effect of particle loss and energy spread}

Energy spread and particle loss, acting separately, destabilize the system, partially or completely unless $\left(-\Gamma^{2}\right)>0$ and $\omega_{\kappa h}>0$. However, acting together, they can expand the region of stability compared with the case when one of the effects is absent.

To illustrate this effect analytically, let us examine the large $\nu_{*}$ limit and small $\mu$ limit, $\mu \ll \omega_{\kappa h} / \Omega_{h}$. If in addition we assume $\omega_{1,2} \ll \omega_{\kappa h}$, the dispersion relation (61) can be reduced to the form

$$
\omega^{2}+\Gamma^{2}+2 a_{*}\left(\omega+\omega_{*_{i}}+\omega_{*_{h}} \mu\right) \omega=-i \mu \Omega_{h} \omega^{2} \gamma / \omega_{\kappa h}^{2}-2 i a_{*}\left(\omega+\omega_{*_{i}}+\omega_{* h} \mu\right)^{2} \omega / \nu_{*} .
$$

Its approximate solutions is $\omega_{1,2} \approx \omega_{1,2}^{(0)}+i \operatorname{Im} \omega_{1,2}$ with

$$
\begin{aligned}
& \omega_{1,2}^{(0)}=\frac{-\left[a_{*}\left(\omega_{*_{i}}+\omega_{* h} \mu\right)+\mu \Omega_{h} / 2\right] \pm\left\{\left[a_{*}\left(\omega_{*_{i}}+\omega_{* h} \mu\right)+\mu \Omega_{h} / 2\right]^{2}-\left(1+2 a_{*}\right) \Gamma^{2}\right\}^{1 / 2}}{1+2 a_{*}} \\
& \operatorname{Im} \omega_{1,2}=\mp \frac{\left[\mu \Omega_{h}\left(\omega_{1,2}^{(0)}\right)^{2} \gamma / \omega_{\kappa h}^{2}+2 \omega_{1,2}^{(0)} a_{*}\left(\omega_{1,2}^{(0)}+\omega_{* i}+\omega_{* h} \mu\right)^{2} / \nu_{*}\right]\left(1+2 a_{*}\right)}{2\left\{\left[a_{*}\left(\omega_{*_{i}}+\omega_{* h} \mu\right)+\mu \Omega_{h} / 2\right]^{2}-\left(1+2 a_{*}\right) \Gamma^{2}\right\}^{1 / 2}}
\end{aligned}
$$

First term in (75) (proportional to $\gamma$ ) destabilizes the $\omega_{2}$ mode if $\omega_{\kappa h}<0$, it stabilizes the $\omega_{1}$ mode if $\left(-\Gamma^{2}\right)<0$ and $\left.\omega_{\kappa h}<0\right)$; otherwise it disappears as $\gamma=0$. The second term (proportional to $1 / \nu_{*}$ ) destabilizes the $\omega_{1}$ modes if $\left(-\Gamma^{2}\right)<0$; otherwise it has a stabilizing effect. Thus both mode $\omega_{1,2}$ are stable in the upper right quadrant of figures 4-7, the mode $\omega_{1}$ is unstable in the left upper. The lower half of figure 4 needs more careful treatment.

For $\left(-\Gamma^{2}\right)<0, \omega_{\kappa h}<0$, the $\omega_{1}$ mode is stabilized by the $\gamma$ term and destabilized by the $\nu_{*}$ term. The $\omega_{2}$ mode is destabilized by the first term and stabilized by the second term. 
The achievement of simultaneous stability would require

$$
\frac{2 a_{*}\left(\omega_{2}^{(0)}+\omega_{*_{i}}+\omega_{* h} \mu\right)^{2}}{-\omega_{2}}>\frac{\gamma \nu_{*} \mu \Omega_{h}}{\omega_{\kappa h}^{2}}>\frac{2 a_{*}\left(\omega_{1}^{(0)}+\omega_{*_{i}}+\omega_{* h} \mu\right)^{2}}{-\omega_{1}} .
$$

This is an incompatible relationship. Note that as $a_{*} \ll 1$ in this analysis, we typically have $\left|\omega_{1,2}\right| \ll \omega_{* h}$. Then to have stability for both modes we require $-\omega_{2}<-\omega_{1}$, which is not the case. Usually it is the $\omega_{1}$ mode that is most difficult to stabilize, especially when $\Gamma^{2} \rightarrow 0$.

For $\left(-\Gamma^{2}\right)>0, \omega_{\kappa h}<0$, the $\omega_{1}$ mode is stable. The achievement of stability for the $\omega_{2}$ modes requires

$$
\frac{2 a_{*}\left(\omega_{2}^{(0)}+\omega_{*_{i}}+\omega_{* h} \mu\right)^{2}}{-\omega_{2}}>\frac{\gamma \nu_{*} \mu \Omega_{h}}{\omega_{\kappa h}^{2}}
$$

In addition for the precessional mode we find for $\omega_{\kappa h} \gg \Gamma$ and $\mu \Omega_{h}$,

$$
\omega_{3}=\omega_{\kappa h}-i \gamma-i 2 a_{*}\left(\omega_{\kappa h}+\omega_{*_{i}}+\omega_{* h} \mu\right)^{2} \mu \Omega_{h} / \nu_{*} \omega_{\kappa h}\left(1+2 a_{*}\right)^{2} .
$$

Here we see that the thermal spread of hot particles stabilizes the precessional mode, while the particle outflow destabilizes the mode if $\omega_{\kappa h}<0$ (assuming $\left|\omega_{\kappa h}\right|<\omega_{*_{i}}+\omega_{*_{h}} \mu$ ) and stabilizes the mode if $\omega_{\kappa h}>0$. Clearly thermal hot ion spread stabilizes the precessional mode for sufficiently small $\mu$.

In order to find marginal conditions explicitly, we once again consider (61) assuming $\omega$ to be real. Separating the real and imaginary parts of (61) we find that at finite $\nu_{*}$ and $\gamma$ the line $\Gamma^{2}=0$ is marginal for the mode with the frequency $\omega=\omega_{1}$ which tends to 0 as $\Gamma^{2} \rightarrow 0$. Determining the other marginal conditions leads to a cubic equation for the parameter $\mu$ :

$$
\left(\omega+\omega_{*_{i}}+\omega_{* h} \mu\right)^{2}\left\{\gamma \Omega_{h} \omega^{2} \mu+2 a_{*} \nu_{*}\left[\left(\omega-\omega_{\kappa h}\right)^{2}+\gamma^{2}\right]\right\}+\gamma \nu_{*}^{2} \Omega_{h} \omega \mu=0 .
$$

Regarding the parameter $\Gamma^{2}$, it is the single-valued function of $\omega$ and $\mu$ :

$$
\Gamma^{2}=-\omega^{2}-\frac{2 a_{*} \nu_{*}\left(\omega+\omega_{*_{i}}+\omega_{*_{h}} \mu\right)\left\{\left(\omega+\omega_{*_{i}}+\omega_{\star_{h}} \mu\right)\left[\left(\omega-\omega_{\kappa h}\right) \omega_{\kappa h}-\gamma^{2}\right]+\nu_{*} \gamma \omega\right\}}{\gamma\left[\left(\omega+\omega_{*_{i}}+\omega_{*_{h}} \mu\right)^{2}+\nu_{*}^{2}\right]} .
$$

At a given real frequency $\omega(79)$ can have one or three (real) solution for $\mu$. Starting from $\gamma=0$, let us track how the region of stability is modified as $\gamma$ grows. figures 8-12 show the 
marginal stability for different values of $\nu_{*}$ and $\gamma$. For comparison they also show the MHD marginal curves described by the (66). This marginal curve, marked by the abbreviation 'MHD', is identical on all figures 6-12 since it corresponds to $\nu_{*}=\infty$ and $\gamma=0$. The condition $\Gamma^{2}=0$, marginal for the mode with the lowest frequency $\omega_{1}$, does not move. As for the marginal condition $\Omega_{h} \mu / \omega_{\kappa h}=0$, it moves downward, thus increasing the region of stability. For $\gamma \rightarrow 0$ and $\omega-\omega_{\kappa h}=\mathcal{O}(\gamma)$ we have

$$
\Omega_{h} \mu / \omega_{\kappa h} \simeq-2 a_{*} \frac{\nu_{*} \gamma}{\omega_{\kappa h}^{2}}\left[1+\left(\frac{\omega-\omega_{\kappa h}}{\gamma}\right)^{2}\right], \quad \Gamma^{2} \simeq-2 a_{*} \nu_{*} \omega_{\kappa h}\left[\frac{\omega-\omega_{\kappa h}}{\gamma}\right],
$$

the corresponding curve has the label 3 on figure 8 . The "precessional mode", which is marginally stable on the curve 3 , is stable above it. Small tick marks directed towards stable region. The two closely spaced curves on the right half of the same figure is approximately described by (68); splitting of these curves is given by the following equation:

$$
\begin{aligned}
\mu & =-\frac{\omega+\omega_{*_{i}}}{\omega * h} \pm\left[\frac{\left(\omega_{*_{i}}+\omega\right) \omega \Omega_{h} \nu_{*} \gamma}{2 a_{*} \omega_{* h}\left[\left(\omega-\omega_{\kappa h}\right)^{2}+\gamma^{2}\right]}\right]^{1 / 2}, \\
\Gamma^{2} & =-\omega^{2}-\frac{\left(\omega+\omega_{*_{i}}\right)\left(\omega-\omega_{\kappa h}\right) \omega \Omega_{h} \omega_{\kappa h}}{\left[\left(\omega-\omega_{\kappa h}\right)^{2}+\gamma^{2}\right] \omega_{* h}},
\end{aligned}
$$

where $\omega<-w_{*_{1}}$. In the region, lying between the branches (81), there is an unstable mode; though it is not very important as in this region there already exists an unstable mode for which the marginal condition is given by (81). The left part of the curve 3 , see figure 8 , moves downwards as $\gamma$ grows, see figure 9--12. Finally, we see in figure 12 that it merges with the other marginal condition $\Gamma^{2}=0$, and then they cease to be marginal, see (70).

\section{Conclusions}

In this paper we have obtained a quadratic form which is correct when the Larmor radius is the size of the plasma. It is clear from the structure of the quadratic form that if there is no end loss, then the $m=1$ mode typically would not depend on Larmor radius effects 
if $\omega / \omega_{\kappa h} \gg 1$, as then the rigid displacement mode is an acceptable solution, for which the response is independent of orbit size. However, with end loss term present, the displacement mode perturbation is not an acceptable eigenfunction, and the response for the hot particles will then depend on the Larmor radius. We have described this effect when the Larmor radius is small. Additional calculations are needed to describe the response when the hot particle radius is comparable with the plasma size.

Our theory was limited to electrostatic perturbations. Finite beta effects are expected to have some important effect on the response of the plasma. The electrostatic approximation is expected to be valid when $\partial \beta_{h} / \partial r<\kappa_{h}$ and $\partial \beta_{c} / \partial r<\kappa_{c}$ where $\kappa_{h}$ and $\kappa_{c}$ are the effective curvatures of the hot and background species. For the case where $\partial \beta_{h} / \partial r>\kappa_{h}$, the case treated in Berk and Wong (1987), the precessional mode is present but does not produce a hot particle resonance because the particle drifts due the $\operatorname{grad}-B$, when $\left|\partial \beta_{h} / \partial r\right| \gg\left|\kappa_{h}\right|$, is much larger than the precessional frequency, which is close to the curvature drift frequency. Further, if $\partial \beta_{c} / \partial r>\kappa_{c}$, the system does not have precessional mode response (Berk and Wong 1987). The appropriate equations in this limit, when the Larmor radius is small, has been derived by Newcomb (1973). In this limit the MHD response for the hot and background particle is relatively simple as the response does not contain any particle resonances in the paraxial limit. The finite Larmor radius has to be treated for both hot and background plasmas. To these equations one needs to add the effect of particle end loss. These effects will be analyzed in another paper (Berk and Kotelnikov 1993).

\section{REFERENCES}

ANIKEEV, A.A., BAGRYANSKY, P.A., IVANOV, A.A., KUZMIN, S.V. SALIKOVA, T.V. 1992 Plasma Physics and Controlled Fusion 34, 1185.

ANIKEEV, A.A., et al., International Conference on Open Plasma Confinement System for Fusion, June 14-18, 1993, Novosibirsk, Russia. 
BERK, H.L., ROSENBLUTH, M.N., VAN DAM, J., SPONG, D. 1983 Phys.

Fluids 26, 201.

BERK, H.L., WONG, H.V., TSANG, K.T. 1987 Phys. Fluids 30, 2681.

BERK, H.L., KOTELNIKOV, I.A., submitted to Phys. Letts.

BERK, H.L., RYUTOV, D.D., TSIDULKO, YU.A. 1991 Phys. Fluids B 3, 1346.

COHEN, B.I., FREIS, R.P., NEWCOMB, W.A. 1986. Phys. Fluids 29, 15581577.

DOMINGUEZ, R.R., BERK, H.L. 1984 Phys. Fluids 27, 1142-1148.

GREENE, J.M., COPPI, B. 1965 Phys. Fluids 8, 1745.

KRALL, N.A. 1986 Phys. Fluids 9, 820.

LIU, J., HORTON, W., SEDLAK, J.E. 1987 Phys. Fluids 30, 467.

MIKHAYlOVSKAYA, L.V. 1984 Sov. J. Plasma Physics 10, 345.

NEWCOMB, W.A. 1973 Ann. Phys. 81, 231. ROSENBLUTH, M.N., LONG-

MIRE, C.L. 1957 Annals of Physics 1, 120.

ROSENBLUTH, M.N., KRALL, N.A., ROSTOKER, N. 1962 Nuclear Fusion

Suppl. Pt. 1, 143-150.

TIMOFEEV, A.V. 1979 JETPh Lett. 29, 227.

WONG, H.V., ROSENBLUTH, M.N., BERK, H.L. 1989 Phys. Fluids B 1, 826839. 


\section{Figure Captions}

1. Sketch of the plasma density profile near the limiter's edge.

2. Growth rate of the lowest frequency mode $\omega_{1}$ versus $\nu_{*}: a_{*}=0.3,1-\omega_{*_{i}} / \Gamma=0$, $2-\omega_{*_{i}} / \Gamma=4,3-\omega_{*_{i}} / \Gamma=8$.

3. MHD marginal stability curves for rigid mode in two-component plasma;

$\nu_{*}=0$. Stable region is towards the upper right of the solid line. When taking into account finite particle loss rate, $\nu_{*} \neq 0$, stable regions shrink to the quadrant $\left(-\Gamma^{2} / \omega_{\kappa h}^{2}>0, \quad \mu \Omega_{h} / \omega_{\kappa h}>0\right)$. When finite energy spread is accounted for, $\gamma \neq 0$ while $\nu_{*}=0$, absolutely stable region is to the right of the MHD marginal curve in the second quadrant and above $\mu \Omega_{h} / \omega_{\kappa h}=0$.

4. MHD marginal stability for two-cumponent plasma with different Larmor radius for hot ions:

1 - rigid mode, $a_{*}=0 ; 2-5$ stabilized mode, $a_{*}=0.3, \omega_{*_{i}} /\left|\omega_{\kappa h}\right|=0.0 ; 2-\omega_{*_{h}} / \Omega_{h} \equiv$ $\left(\rho_{h} / r_{0}\right)^{2}=0.25 ; 3-\omega_{* h} / \Omega_{h}=0.50 ; 4-\omega_{* h} / \Omega_{h}=0.75 ; 5-\omega_{* h} / \Omega_{h}=1.00 ;$

Stable region is towards the right and upward from the solid line. When taking into account finite outgoing current, i.e. $\nu_{*}$ finite, the stable regions shrinks to the quadrant $\left(-\Gamma^{2} / \omega_{\kappa h}^{2}>0, \quad \mu \Omega_{h} / \omega_{\kappa h}>0\right)$. When finite energy spread is accounted for, $\gamma \neq 0$ while $\nu_{*}=\infty$, absolutely stable region is to the right of the MHD marginal curve in the second quadrant and above $\mu \Omega_{h} / \omega_{\kappa h}=0$.

5. Same plots as on figure 4 in a finer scale.

6. Same plots as on figure 4 except for $\omega_{*_{i}} /\left|\omega_{\kappa h}\right|=1.0$

7. Same plots as on figure 6 in a finer scale. 
8. Marginal stability in two-component plasma for $\nu_{*} /\left|\omega_{*_{h}}\right|=10, \gamma /\left|\omega_{* h}\right|=0.01$. Other parameters are: $a_{*}=0.3, \omega_{* h} / \Omega_{h}=0.5, \omega_{*_{i}} / \omega_{\kappa h}=1$. The curve marked as 'MHD' is MHD marginal condition, the one marked by 1 is marginal for the $\omega_{1}$ mode, etc. The tick marks are directed towards the region stable against corresponding mode. The absolutely stable region is to the right of the $\omega_{1}$ nuarginal line and above the $\omega_{3}$ marginal line in the fourth quadrant.

9. Marginal stability in two-component plasma for $\nu_{*} /\left|\omega_{\kappa h}\right|=5, \gamma /\left|\omega_{\kappa h}\right|=0.1$. Other parameters are as in figure 8.

10. Same plots as in figure 9 in a finer scale.

11. Marginal stability in two-component plasma for $\nu * /\left|\omega_{\kappa h}\right|=1, \gamma /\left|\omega_{\kappa h}\right|=0.1$. Other parameters are as in figl: : 8.

12. Marginal stability in two-component plasma for $\nu_{*} /\left|\omega_{\kappa h}\right|=0.2, \gamma /\left|\omega_{\kappa h}\right|=0.2$. Other parameters are as in figure 8. 


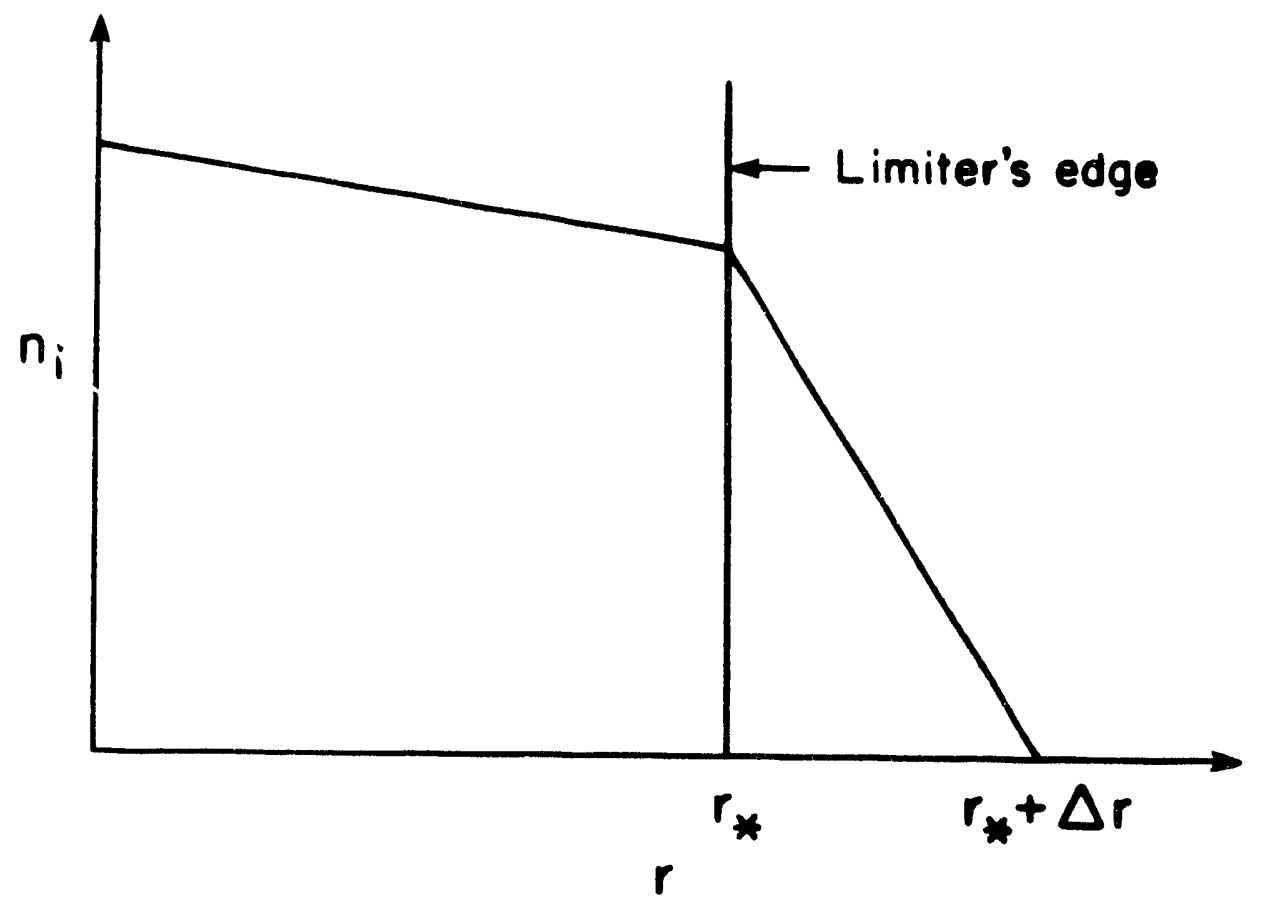




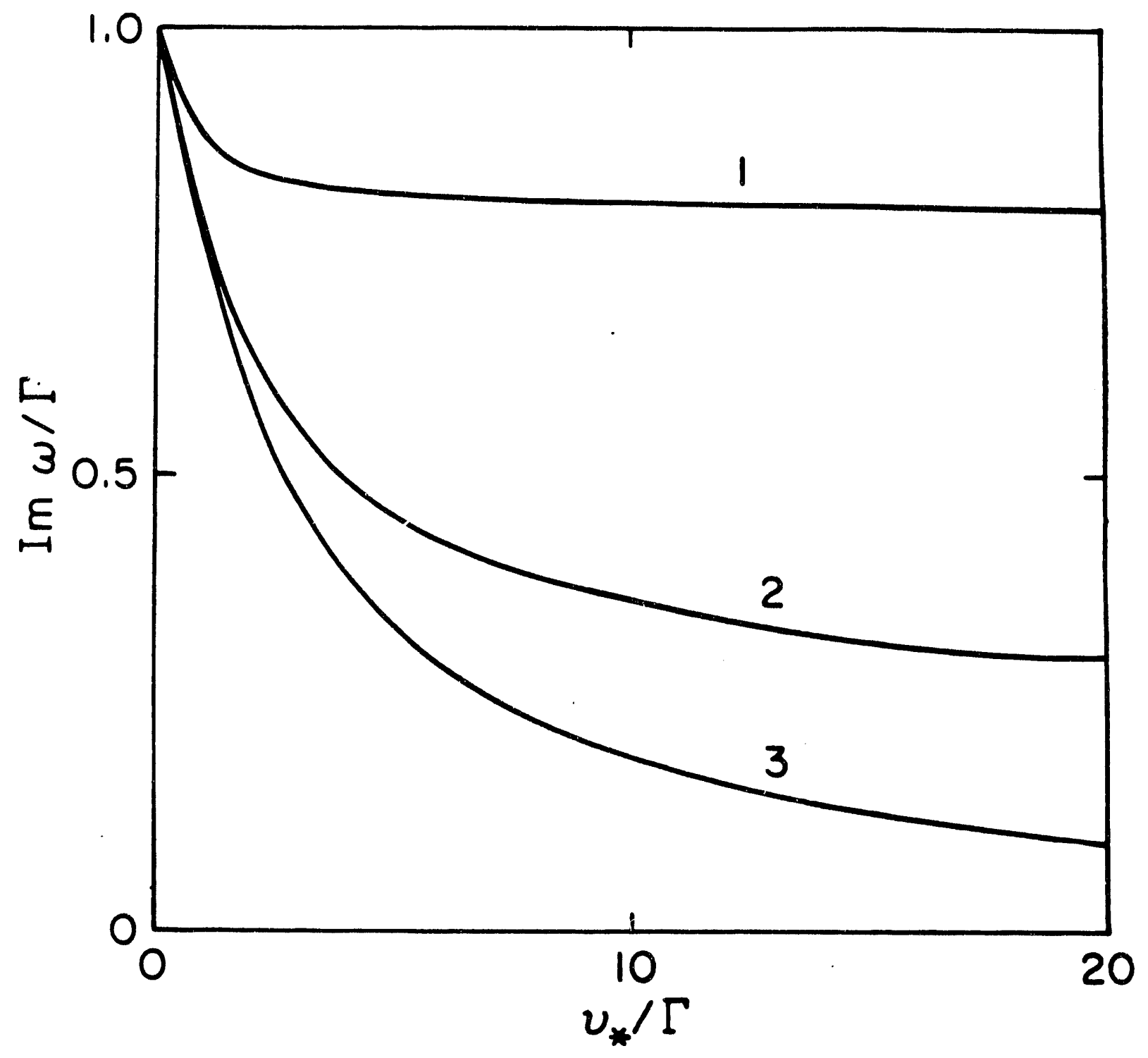

2 


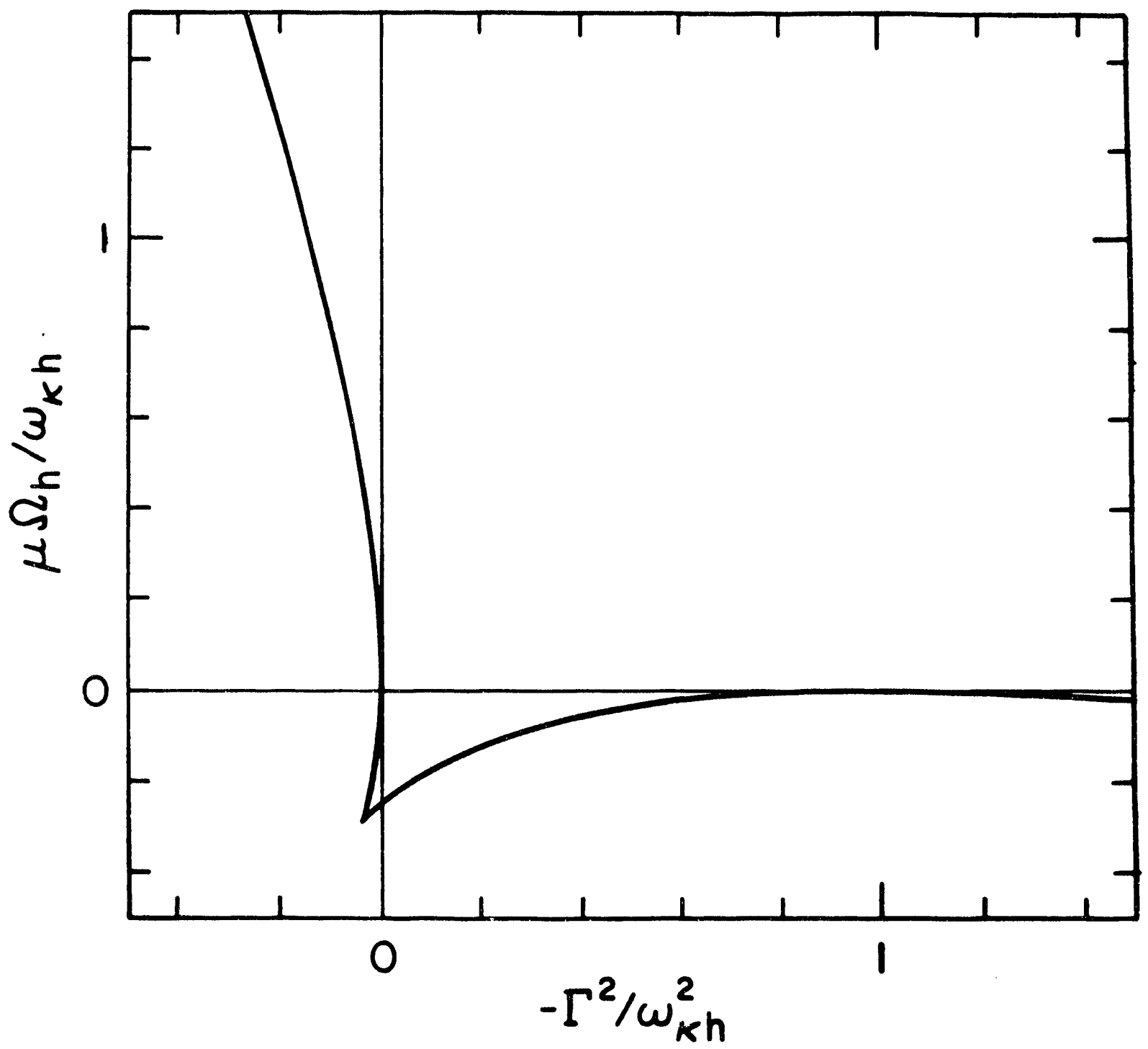




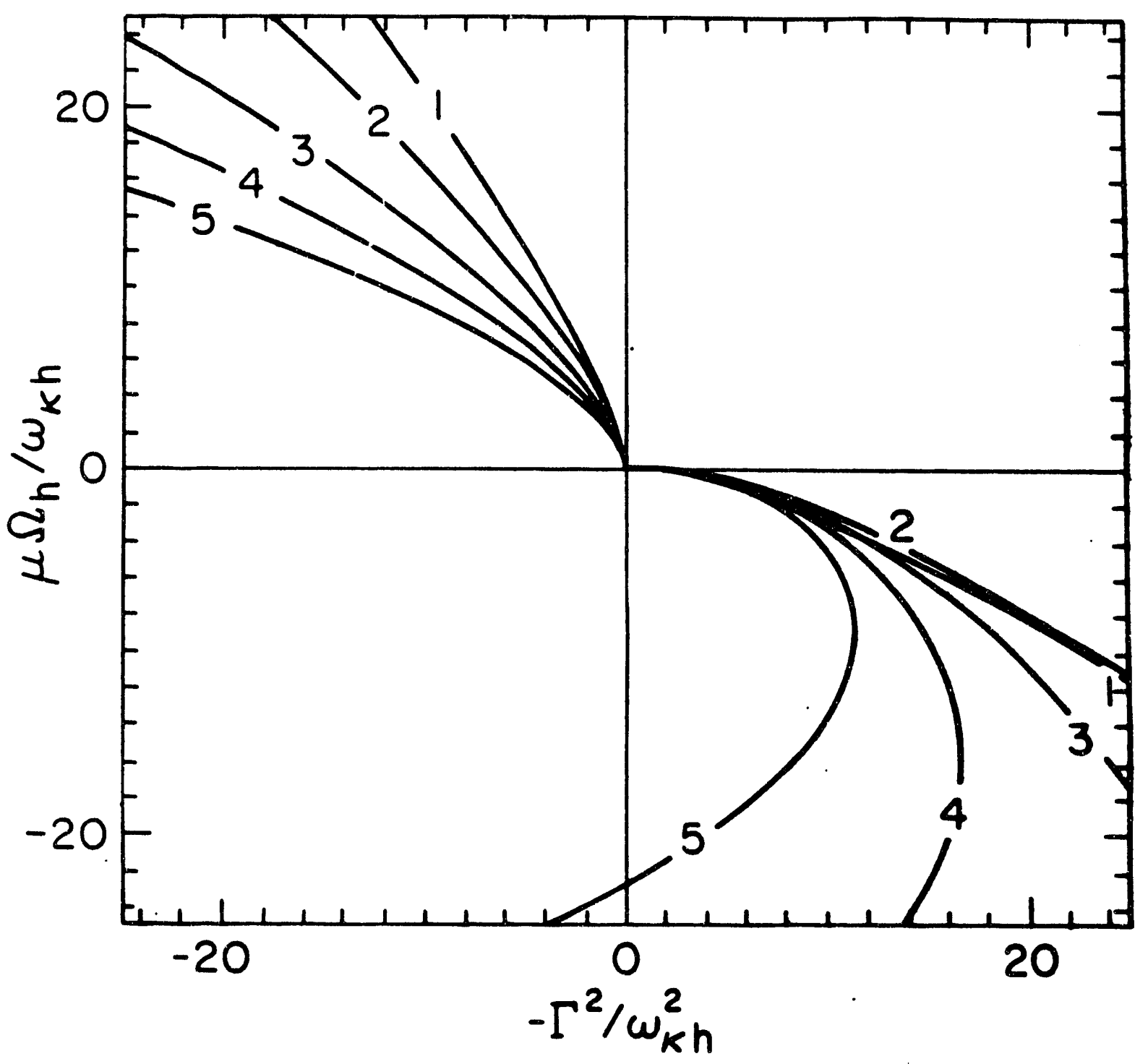




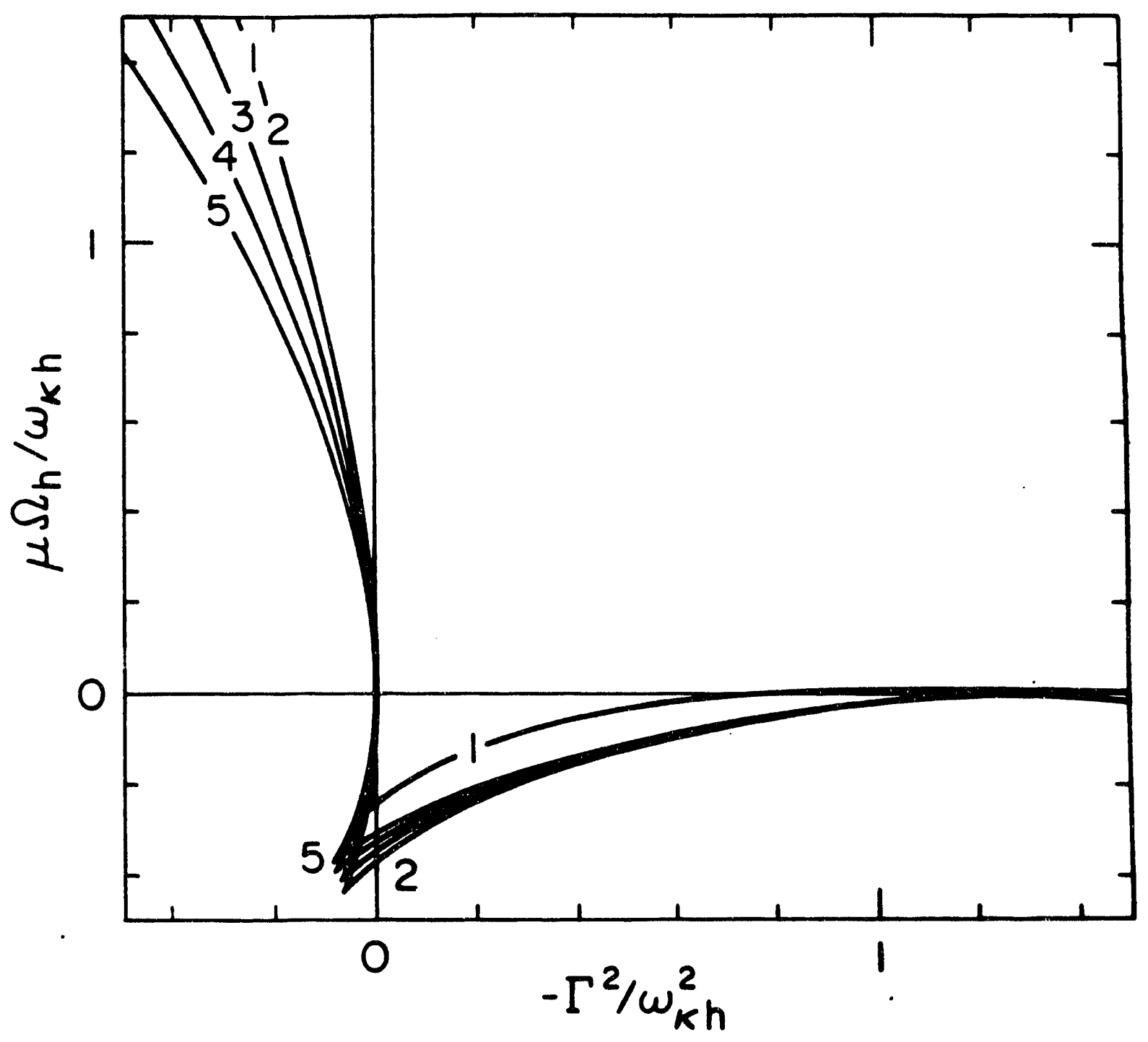




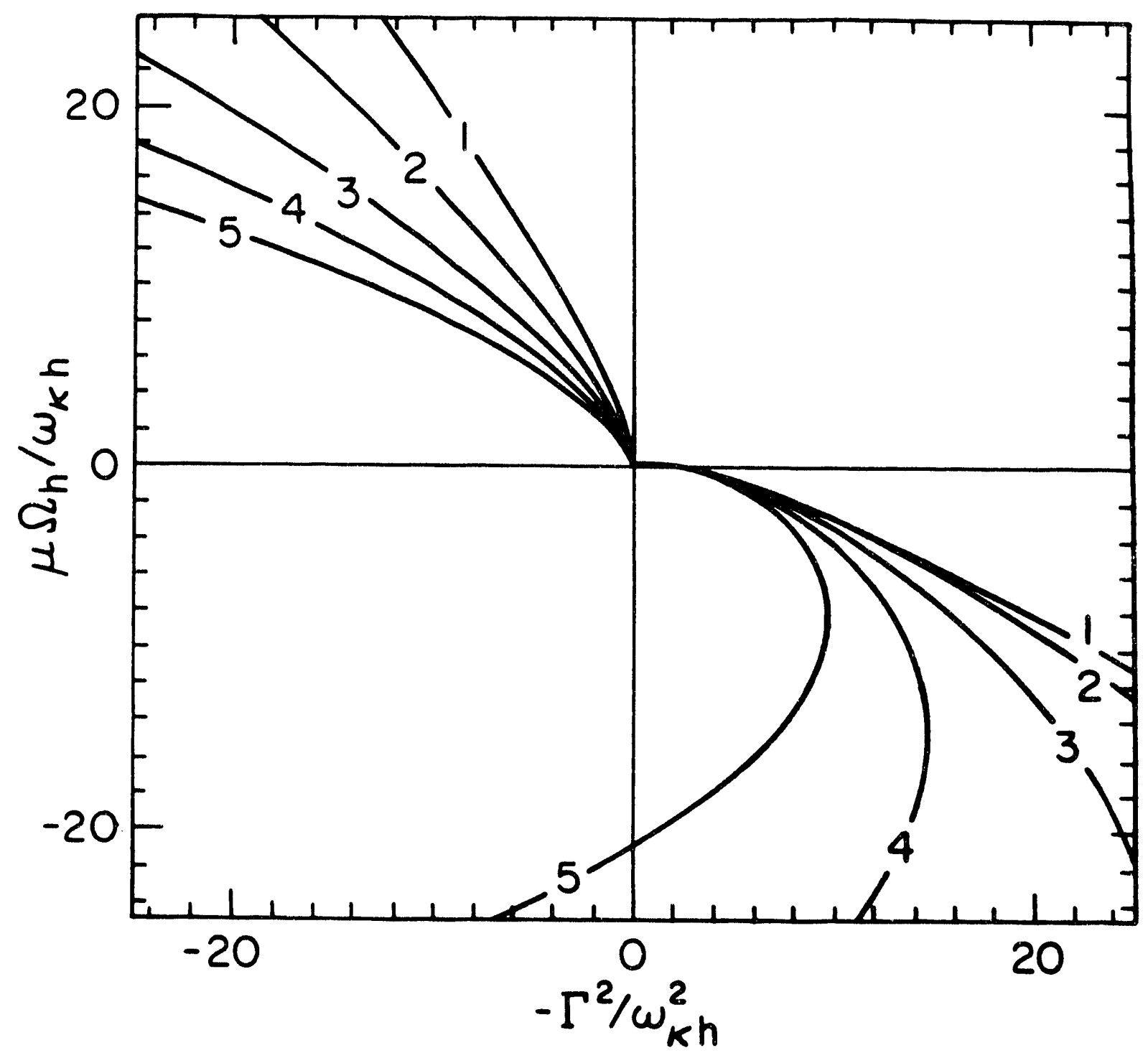




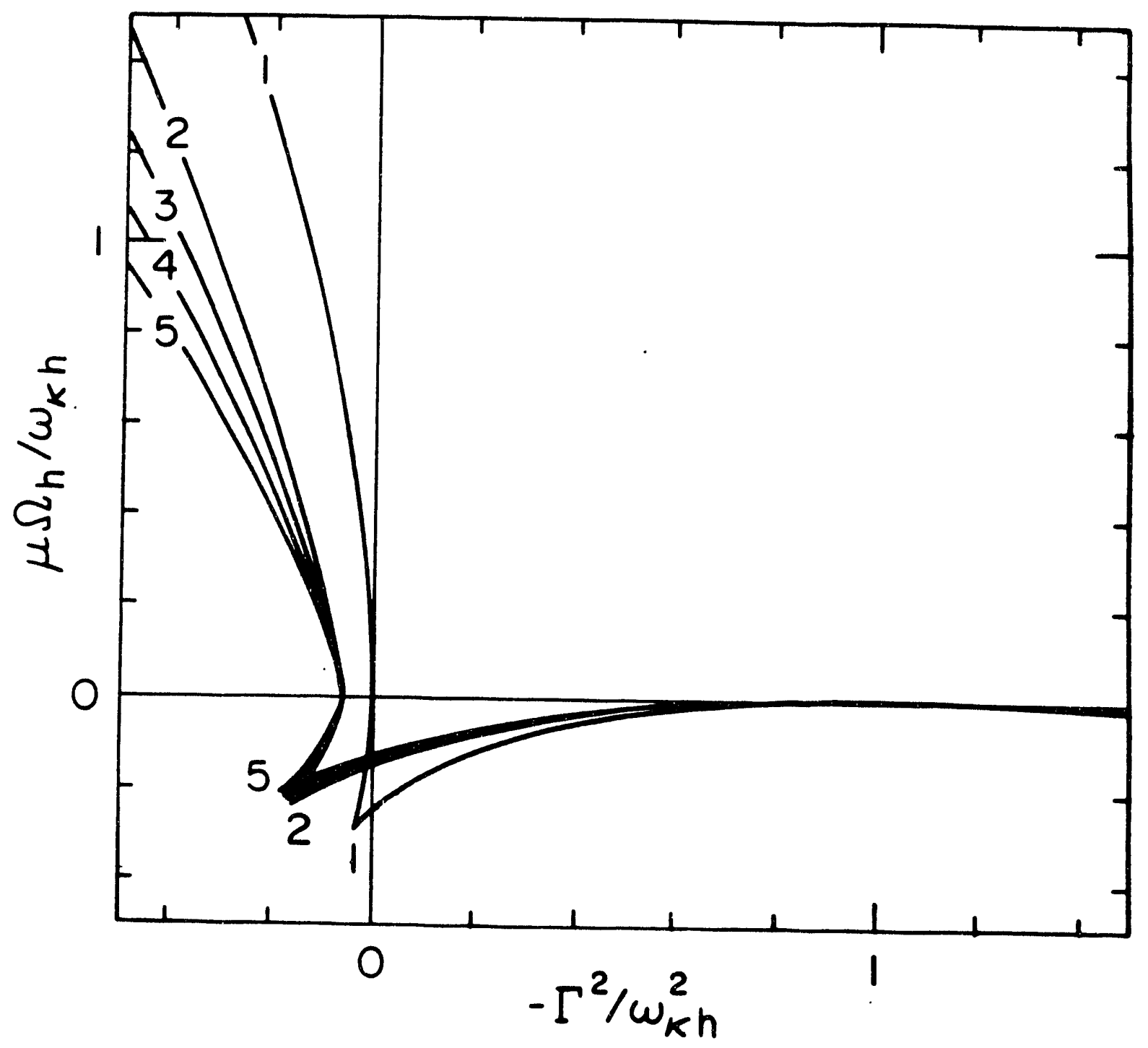




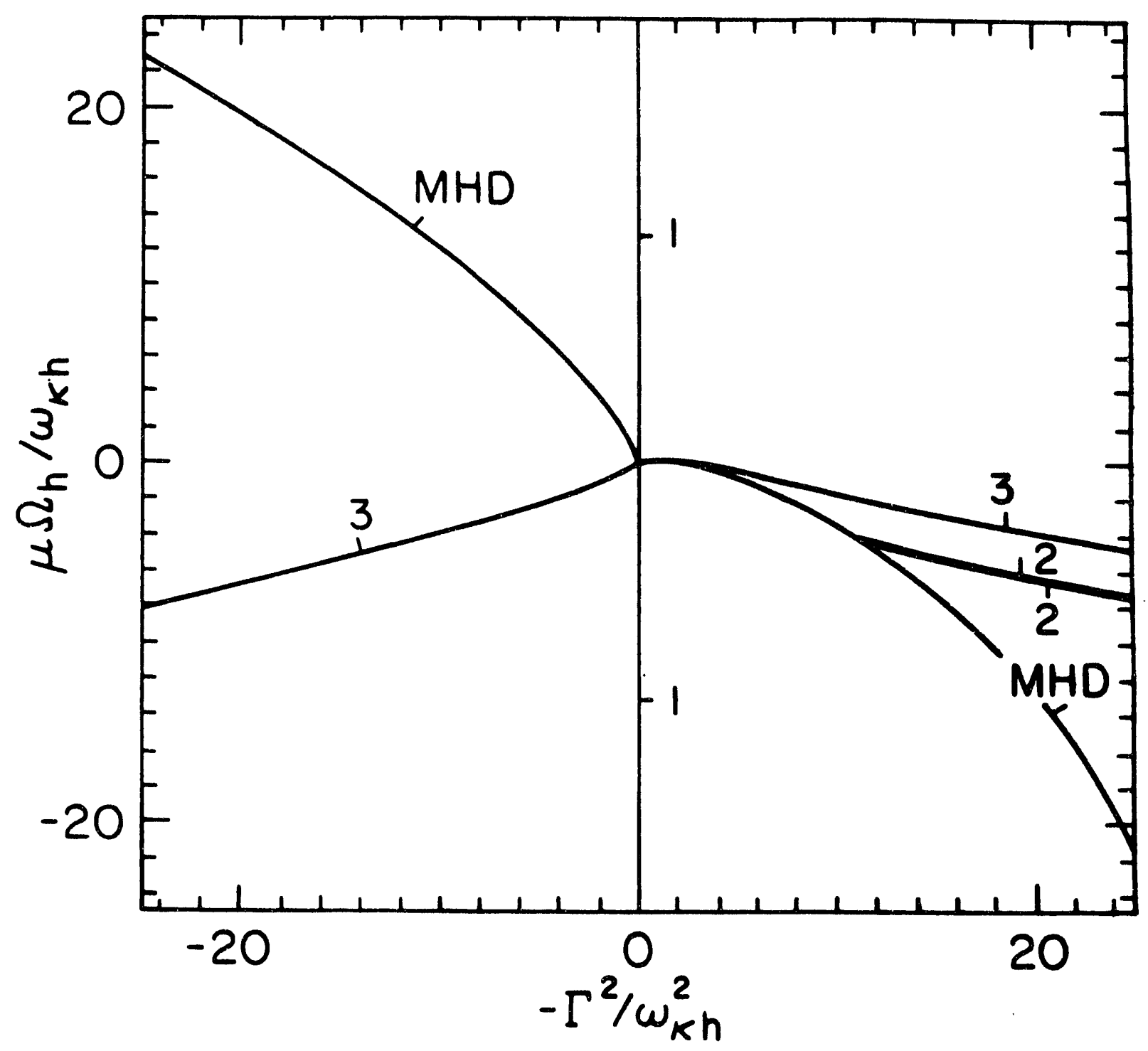




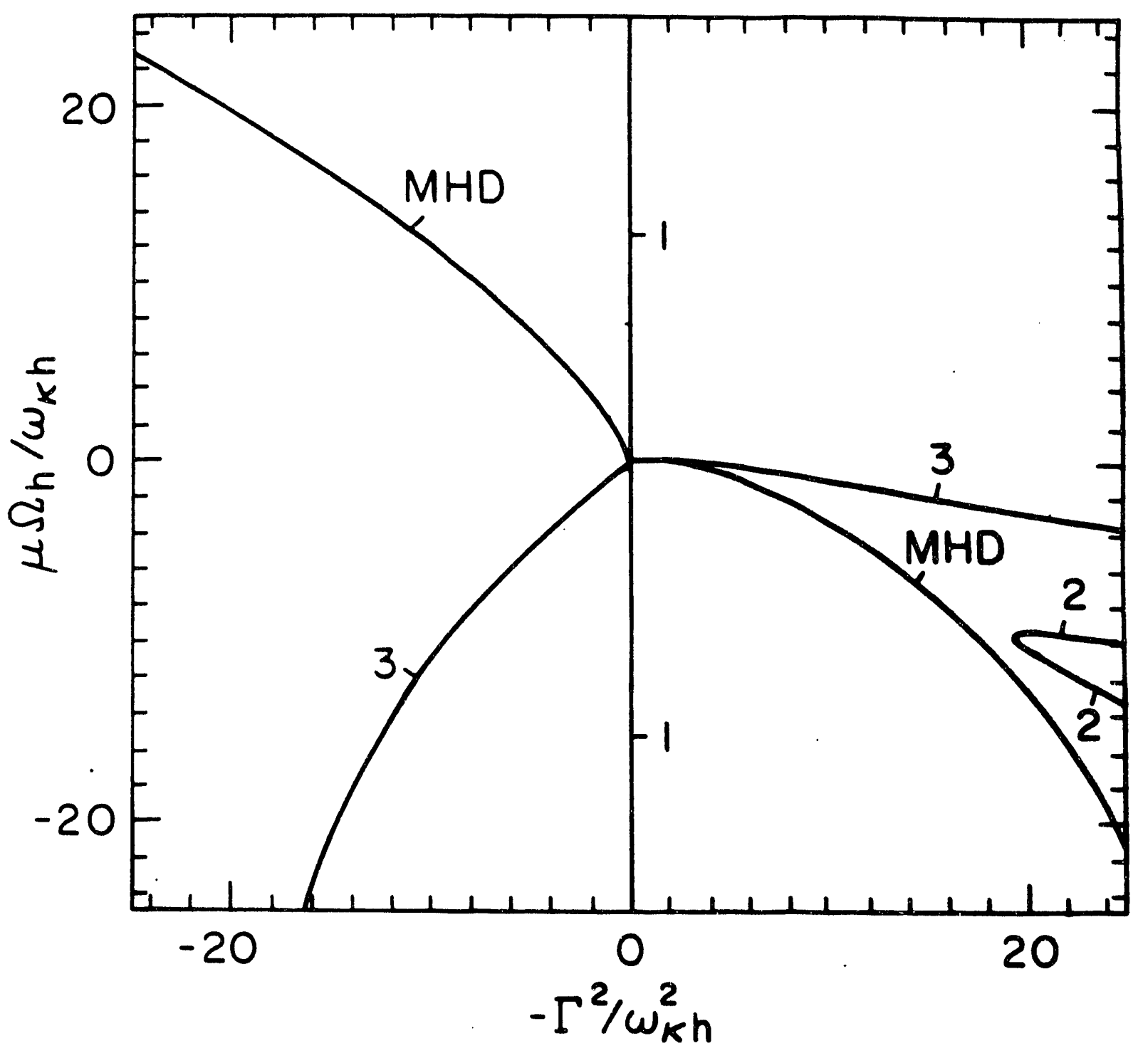

9 


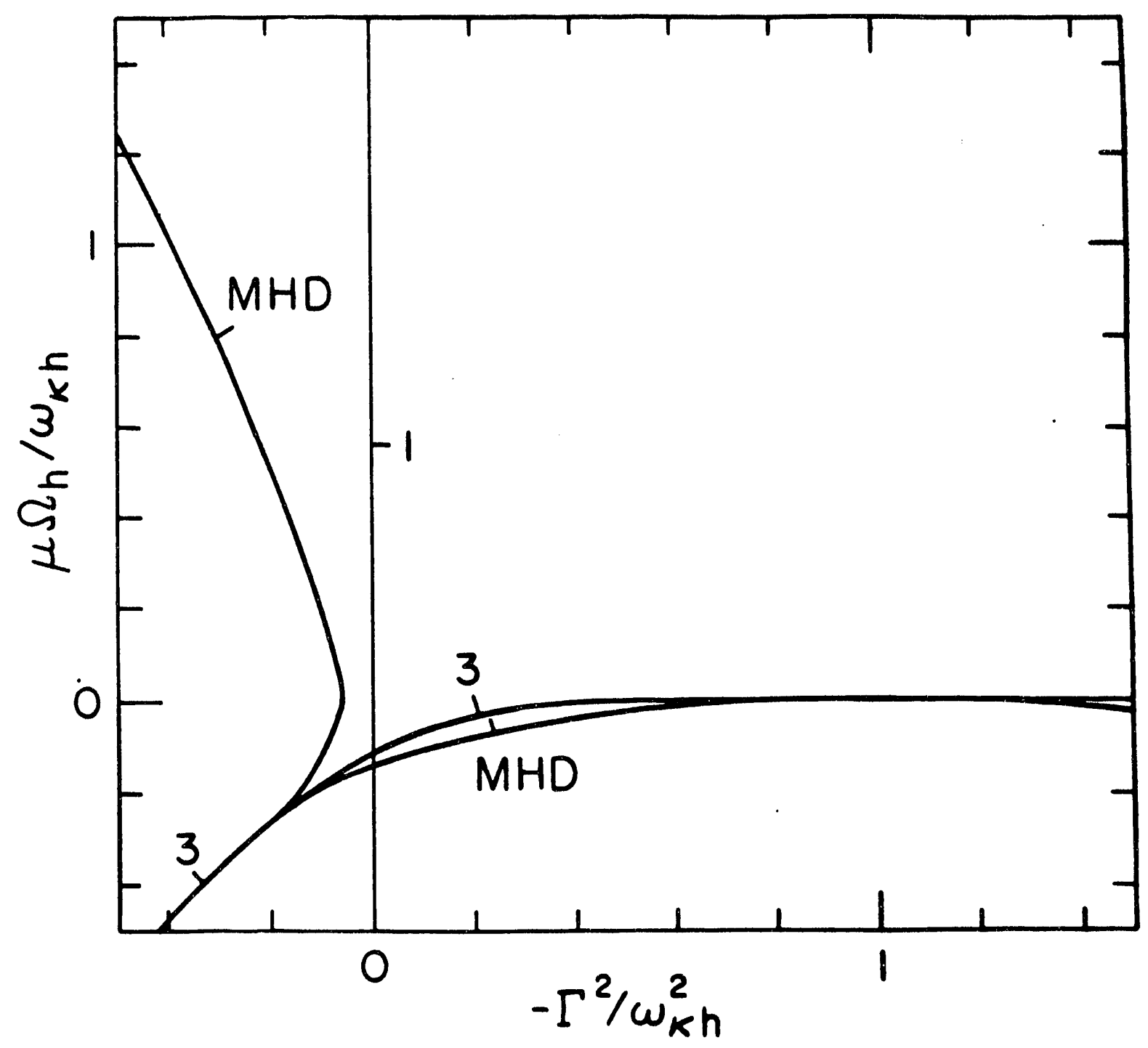




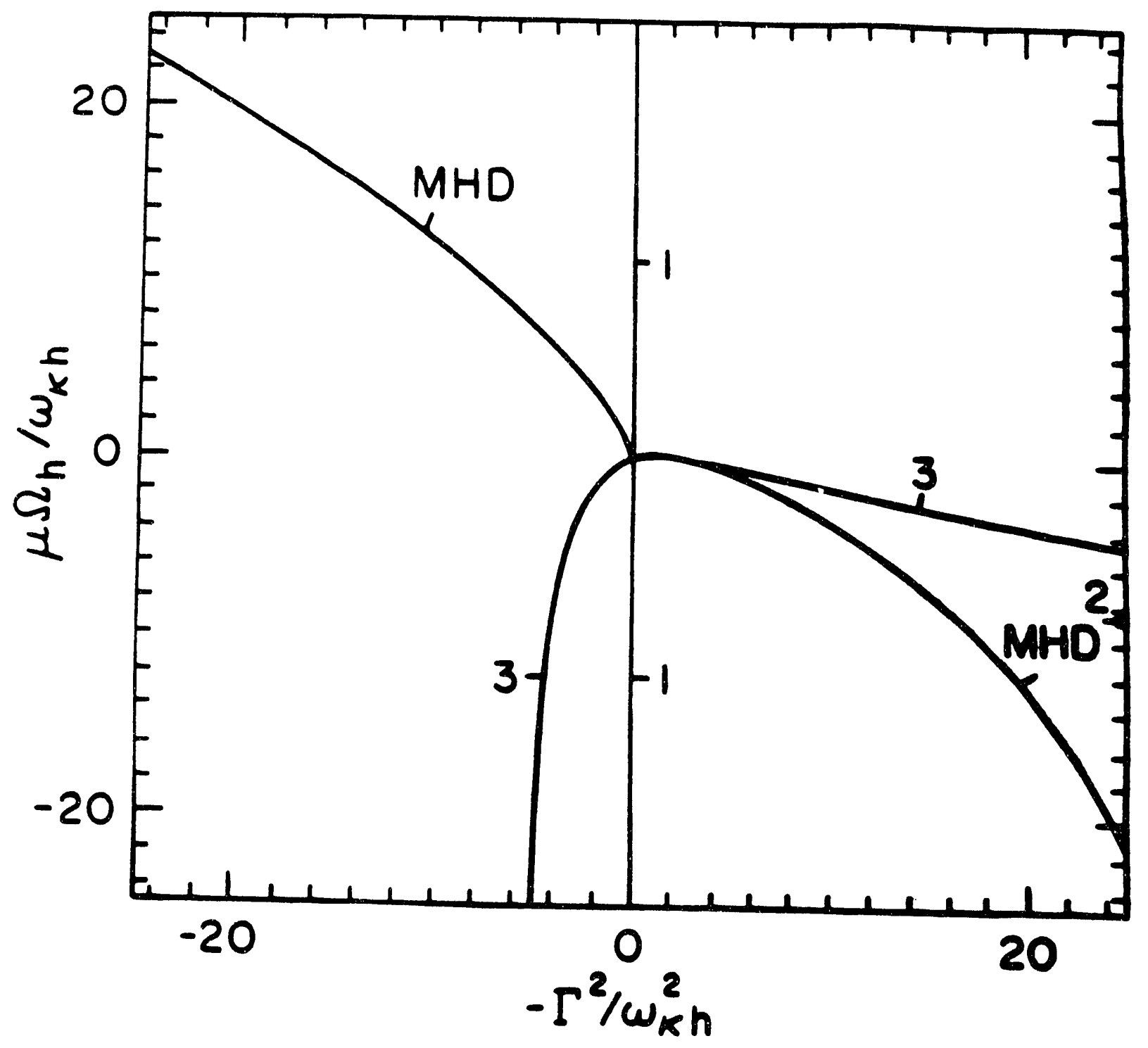




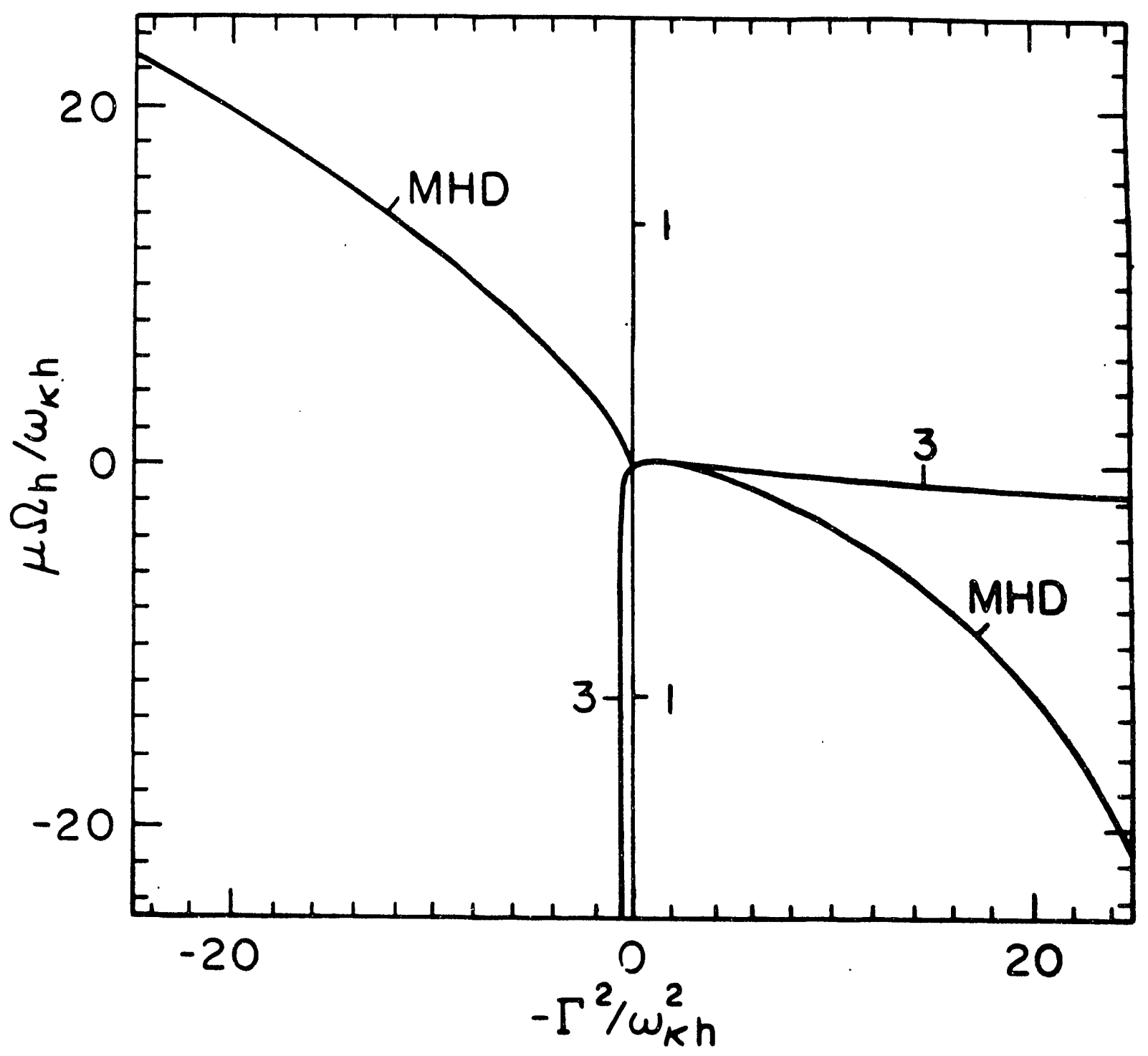

R 

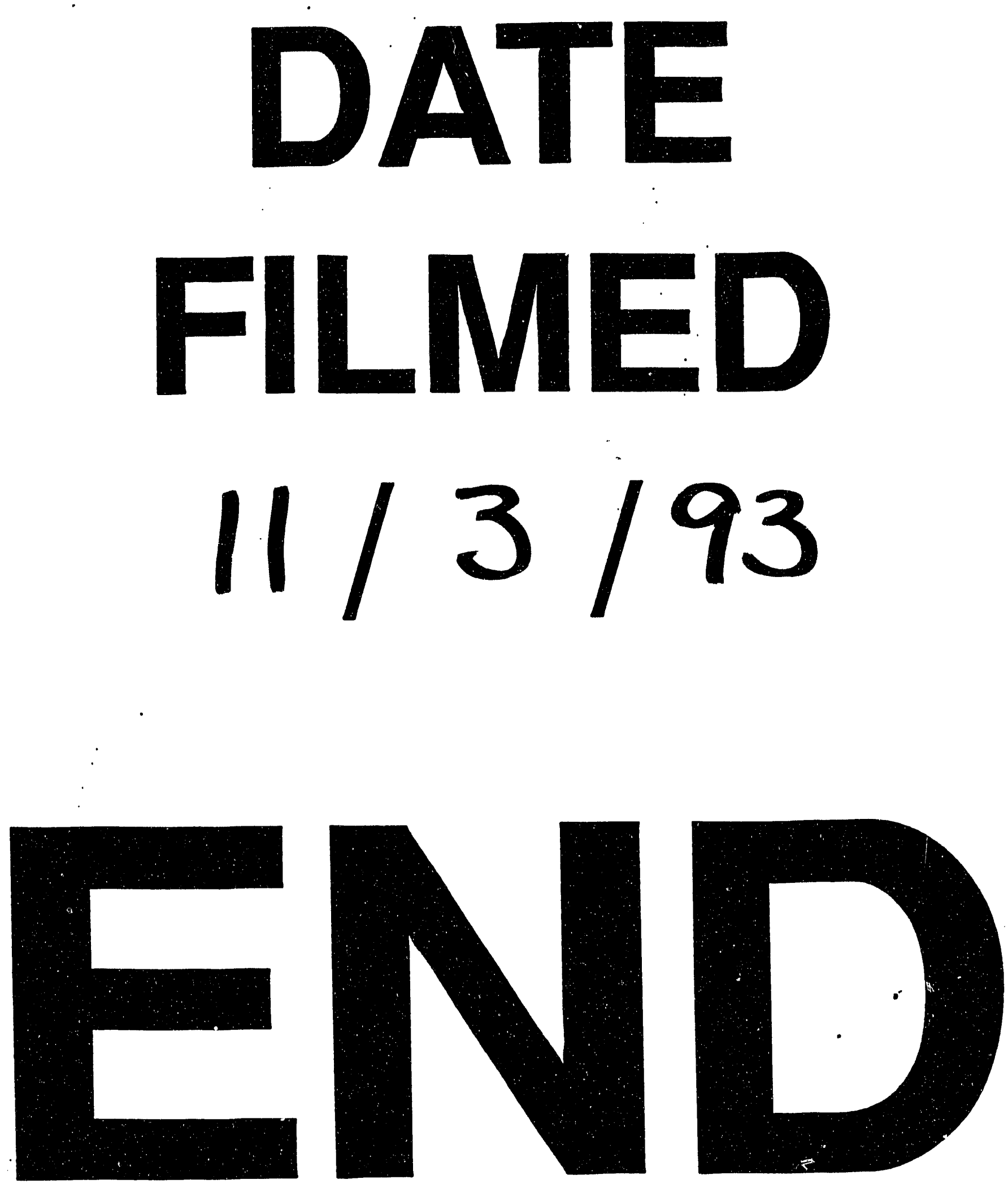